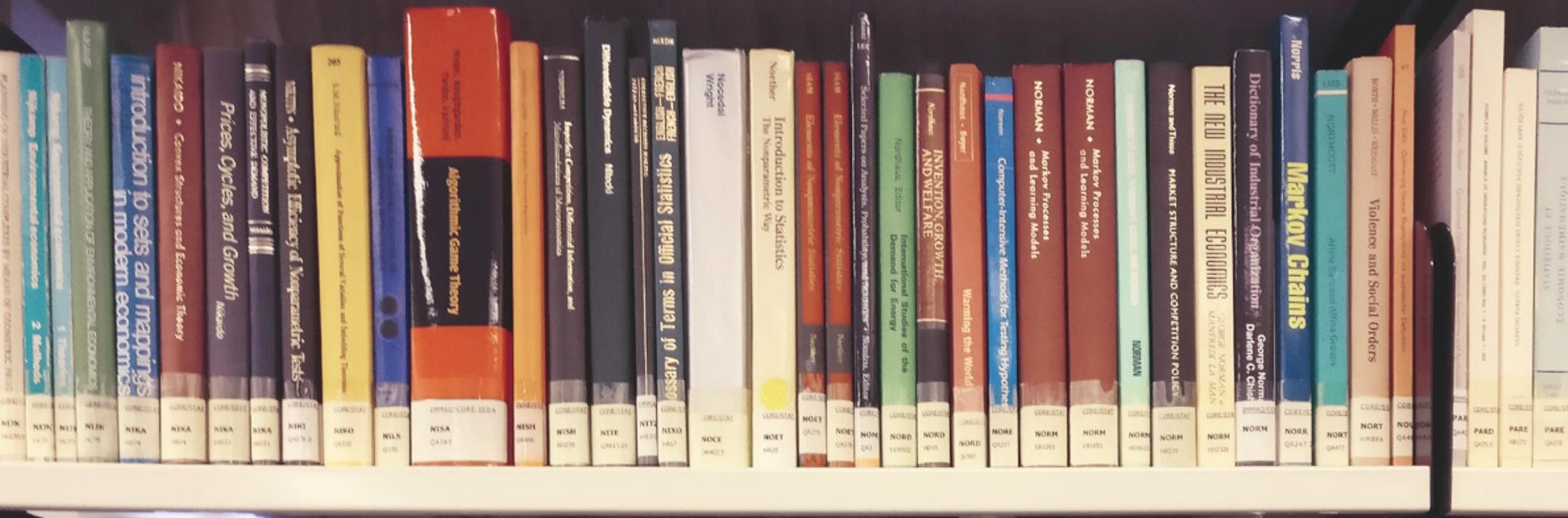

$2017 / 22$

DP

Ana Mauleon, Nils Roehl and Vincent Vannetelbosch

Constitutions and groups

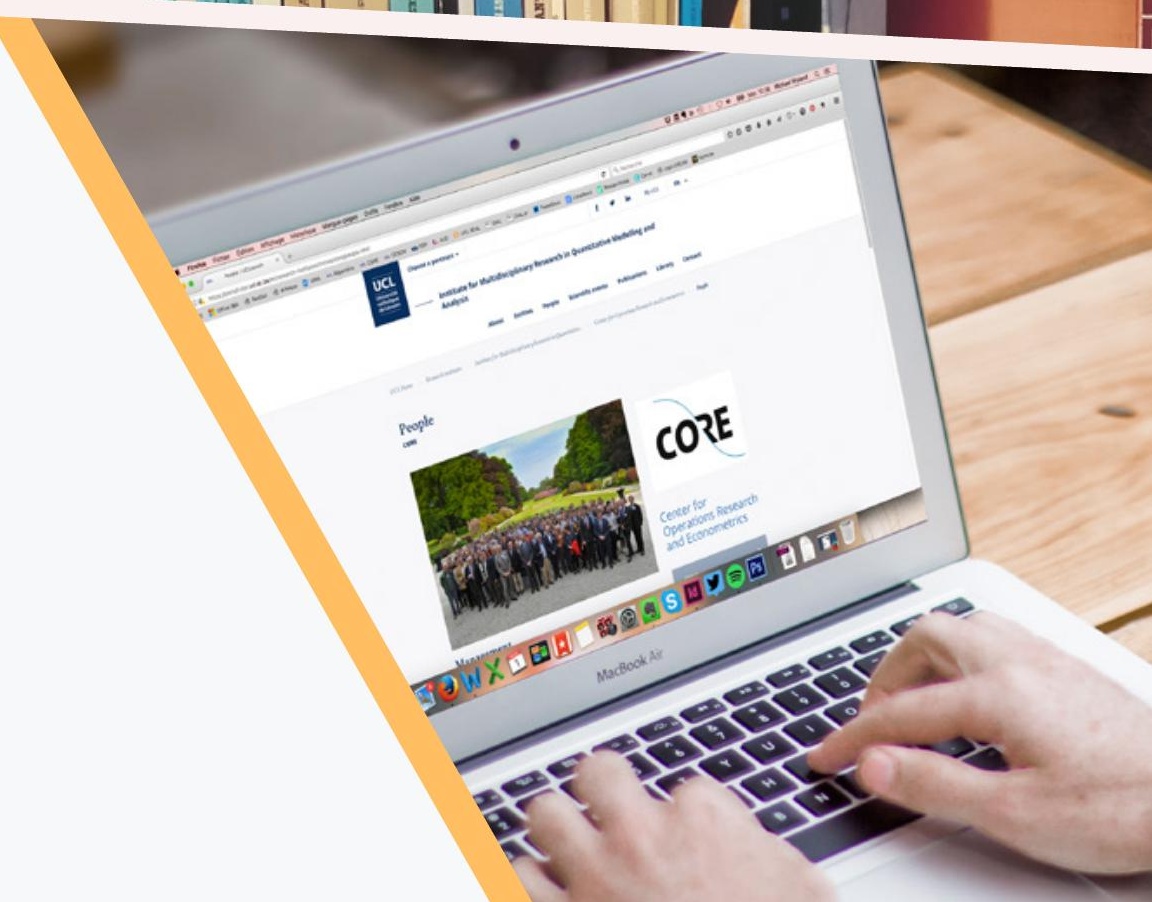




\section{CORE}

Voie du Roman Pays 34, L1.03.01

Tel (32 10) 474304

Fax (32 10) 474301

Email: immaq-library@uclouvain.be

https://uclouvain.be/en/research-institutes/

immaq/core/discussion-papers.html 


\title{
Constitutions and Groups
}

\author{
Ana Mauleon* Nils Roehl ${ }^{\dagger} \quad$ Vincent Vannetelbosch ${ }^{\ddagger}$
}

August 25, 2017

\begin{abstract}
We develop a general theoretical framework that allows us to study the group structures that are going to emerge at equilibrium when individuals are allowed to engage in several groups at the same time. We introduce the notion of constitution in order to model for each group the rules governing both the composition of the group and the conditions needed to leave the group and/or to become a new member of the group. We then propose the concept of constitutional stability to study the group structures that are going to emerge at equilibrium in overlapping coalition settings. This concept generalizes previous stability concepts in the literature in which the constitutional rules were exogenously given or not explicitly considered. We combine requirements on constitutions and preferences for guaranteeing both the existence and the emergence of constitutionally stable group structures. Furthermore, by embedding many-to-many matchings into our setting, we show how these results could be useful to identify the constitutionally stable group structures in this particular environment.
\end{abstract}

Key words: Overlapping Coalitions, Group structures, Constitutions, Stability, Many-to-Many Matchings.

JEL classification: C72, C78, D85.

\footnotetext{
${ }^{*}$ CEREC, Saint-Louis University - Brussels; CORE, University of Louvain, Louvain-la-Neuve, Belgium. E-mail: ana.mauleon@usaintlouis.be

${ }^{\dagger}$ Department of Economics, University of Paderborn, Paderborn, Germany. E-mail: nils@famroehl.de

${ }^{\ddagger}$ CORE, University of Louvain, Louvain-la-Neuve; CEREC, Saint-Louis University - Brussels, Belgium. E-mail: vincent.vannetelbosch@uclouvain.be
} 


\section{Introduction}

1.1. Overview. Many economic, social and political activities are conducted by groups or coalitions of individuals. For example, consumption takes place within households; production is carried out by firms which can be seen as large coalitions of owners of different factors of production; firms pool their expertise in joint ventures and strategic alliances; workers are organized in unions or professional associations; political life is conducted through political parties and interest groups; and individuals belong to formal and informal social clubs. The understanding of how and why such groups form and the precise way in which they affect outcomes of social and economic interactions has been apprehended assuming that each individual can only be member of one of these groups. ${ }^{1}$ However, there are many situations in which individuals might be member of more than one group. Firms A, B and C might cooperate in a strategic alliance, firms B and D might cooperate in another alliance, while firms A and D may not cooperate with each other. Overlapping groups of individuals may be involved in relationships involving public-goods provision, reciprocity or information-sharing. Free trade agreements are signed among overlapping collections of countries.

Up to now, very little theoretical work exists on overlapping coalition formation settings. Chalkiadakis et al. (2010) introduce a model for cooperative games with overlapping coalitions that is applicable in situations where agents need to allocate different parts of their resources to simultaneously serve different tasks as members of different coalitions. They explore the stability concept of the core ${ }^{2}$ in such overlapping coalition setting. ${ }^{3}$ The goal of our paper is to provide a general theoretical framework that could be used to study the stability of any situation involving overlapping coalitions, and not only of specific cooperative or non-cooperative games with overlapping coalitions. It departs from previous work in two important aspects. First, in order to be as general as possible, we abstract from activities carried out within each group and we suppose that individuals' preferences depend on the group structure. Second, we introduce the notion of constitution to model for each group the rules governing both the composition of the group (restricting the set of feasible deviating coalitions) and the conditions needed to leave the group and/or to become a new member of the group (i.e., the supporting coalitions that could grant the admission into the group of each feasible deviating coalition). We want the rules

\footnotetext{
${ }^{1}$ Ray (2007) and Ray and Vohra (2015) provide surveys of models of coalition formation.

${ }^{2}$ Core stability implies that no group of agents should be able to profitably deviate from a configuration in the core.

${ }^{3}$ See also the work of Myerson (1980), Shenoy and Kraus (1996), Dang et al. (2006), Conconi and Perroni (2002) and Albizuri et al. (2006) for other specific cooperative or non-cooperative models of overlapping coalitions.
} 
or constitutions to be as general as possible in order to capture a wide spectrum of possible applications. For instance, some groups could have capacity constraints or some legal requirements regarding the type of member that could be part of the group. In some groups it might be possible to dismiss members but in others there might be a protection against dismissal. Or, in some groups entry might be free but in others it might require the consent of certain number of members (a majority or the unanimity of them, for example). Therefore, both the individuals' preferences and the constitutional design may have a significant impact on the formation and stability of group structures.

We then propose the concept of constitutional stability to predict the group structures that are going to emerge at equilibrium in overlapping coalition settings where the deviating coalition has to take into account the constitution of the group she wants to modify. This concept generalizes previous stability concepts in the literature in which the rules governing the composition of each group as well as the exit of current members and/or the arrival of new members were exogenously given or not explicitly considered. ${ }^{4}$ The idea of constitutional stability is that modifying the composition of a group (according to its constitution) via the deviation of a feasible deviating coalition needs the consent of both the deviating players and every member of at least one of the supporting coalitions that could grant the admission into the group and/or the departure of the group of such feasible deviating coalition. The other main contribution of this paper is exploring the stability concept of constitutional stability in two different ways. We examine both the existence of constitutionally stable group structures as well as whether the society will reach one of these stable group structures.

To this end, we adapt to our setting the random dynamic process developed for marriage problems by Roth and Vande Vate (1990). They show that the Markov process converges to a stable matching with probability one. In our study, we use the notion of improving path of Jackson and Watts $(2001,2002)$ who propose a dynamic process of network formation in which individuals form and sever links based on the improvement that the resulting network offers them relative to the current network. Jackson and Watts (2002) prove that this deterministic dynamic process may end at a pairwise stable network or may cycle. In our framework, an improving path is a sequence of group structures that can emerge when players join or leave some groups based on the improvement the resulting group structure offers them relative to the current one. Each group structure in the sequence differs from the previous one in that one group is modified by a feasible deviating coalition and

\footnotetext{
${ }^{4}$ The most used stability concept in both the traditional non-overlapping scenario and the overlapping one is the core. Typically, it assumes that the deviators only form coalitions among themselves and, thus, no composition and/or admission rules are considered.
} 
every player joining the group strictly prefers the resulting group structure to the current one. Moreover, the deviation should not be blocked and, hence, there should be a supporting coalition that strictly benefits from the deviation. ${ }^{5}$

We show that the society induces a constitutionally stable group structure if and only if there are no closed cycles. ${ }^{6}$ We provide requirements on constitutions and individuals' preferences guaranteeing that, from every initial group structure, there always exists an improving path leading to a constitutionally stable group structure. In other words, we show the kind of constitutions that leads to stability and we explore relations between various constitutional arrangements and individuals' preferences guaranteeing stability.

One of the most interesting features of our model is its versatile applicability since overlapping groups appear in many environments. The last part of this paper is devoted to a particular application, namely to job markets with labor unions, where we show how the previous results could be useful to identify the constitutionally stable group structures in this particular environment. By embedding many-to-many matchings into our setting, we study the existence of constitutionally stable firm structures (i.e., matchings) in three environments that differ in the level of authority that the owners of the firm and the workers could have. We show that the job market becomes stable if the degree of authority of one side of the market becomes sufficiently high. We also find a variation of Roth's "polarization of interests" (cf. Roth, 1984) between employers and employees: Each side of the market would be worse off if the other side obtains more degree of authority.

1.2. Literature Review. The formation of social groups is of fundamental interest and it has been examined from numerous angles. For instance, Ellickson et al. $(1999,2001)$ as well as Allouch and Wooders (2008) analyze this issue in the context of general equilibrium theory, Acemoglu et al. (2012) provide a dynamic model for studying the stability of societies, and Page and Wooders (2010) formalize club formation as a non-cooperative game, to name but a few. In fact, providing a complete overview over all publications dealing with group formation in a broader sense would exceed the scope of the paper due to the great complexity and diversification of the field. The following survey restricts on most closely related branches and outlines which publications particularly influence our work.

Analyzing group formation but abstracting from activities carried out within each group obviously relates to hedonic coalition formation (e.g., Banerjee et al., 2001; Bogomolnaia and Jackson, 2002). Moreover, studies dealing with economic

\footnotetext{
${ }^{5}$ Our notion of improving path is also a variation of the notion of path in Roth and Vande Vate (1990); i.e., the random sequence of matchings generated from an arbitrary matching by randomly satisfying a blocking pair at each step in the sequence.

${ }^{6}$ This result has been established by Roth and Vande Vate (1990) for marriage problems.
} 
networks (e.g., Jackson, 2008) or with matching markets (e.g., Roth and Sotomayor, 1990) can also be embedded into our setting. Thus, we contribute indirectly to a stream of literature where the authors combine coalition formation and matching problems (e.g., Cesco, 2012; Pycia, 2012). However, the way we model group structures and preferences is closer to models from matching theory where individuals are not only concerned about which groups they belong to but also about who the other members of the groups are (e.g., Dutta and Masso, 1997; Echenique and Yenmez, 2007; Kominers, 2010).

One of the main contributions of this paper is to formalize constitutional rules within a hedonic setting of overlapping groups. This approach is in spirit with some other publications from literature, like Bala and Goyal (2000), Page and Wooders (2009), Jehiel and Scotchmer (2001), Drèze and Greenberg (1980) or Caulier et al. (2013a, 2013b), among others. However, the aforementioned works differ from ours in several important aspects.

Our concept of constitutional stability is related to the concept of contractual stability that has been studied in the literature on coalition and network formation (see Drèze and Greenberg, 1980). The idea of contractual stability is that modifying the coalition structure and/or the network structure needs the consent of both the deviating players and their original coalition partners. Caulier et al. (2013a, 2013b) study the stability of situations where players are part of a network and belong to a coalition in some coalition structure under two different rules for consent: simple majority and unanimity. ${ }^{7}$ It also relates to other stability concepts in the literature that formalize specific constitutional rules. Jehiel and Scotchmer (2001) characterize four equilibrium concepts associated to four constitutional rules of admission in a model of jurisdiction formation: free mobility, admission by majority vote, admission by unanimous consent and admission by a demand threshold for public goods.

Page and Wooders (2009) introduce a model of network formation whose primitives consist of a feasible set of networks, players preferences, rules of network formation and a dominance relation on feasible networks. They characterize the sets of networks that are likely to emerge and persist ${ }^{8}$ by means of the path dominance core. The path dominance core is defined as the set of networks having the property that no network in the set is path dominated by any other feasible network. The path dominance core is non-empty if and only if there is a basin of attraction containing a single network. However, no condition on preferences and/or on the

\footnotetext{
${ }^{7}$ See Mauleon et al. (2016) for a study of how different rules of consent (unanimity and simple majority) for exiting an alliance affect the formation of strategic alliances in Bloch's (1995) model of associations of firms where the existence of overlapping alliances is not allowed.

${ }^{8}$ See Bala and Goyal (2000) for an alternative study of the dynamics of network formation using a version of the best-response dynamics.
} 
rules of network formation guaranteeing the existence of such basin of attraction is provided.

In the present paper, we go beyond the existing literature on coalition and/or network formation in several respects. First, we focus on the formation of overlapping group structures rather than on the formation of networks or coalition structures where each player can only engage in one group. This extension significantly broaden the set of potential applications. Second, by introducing the notion of constitution, we study the stability of these environments by means of the concept of constitutional stability that generalizes previous stability concepts in which the constitutional rules were exogenously given or not explicitly considered. Third, by allowing each group to have its own constitution, we introduce heterogeneity regarding these rules of consent and we provide a systematic treatment of the relationship between various constitutional arrangements and individuals' preferences guaranteeing both the existence and the emergence of constitutionally stable group structures.

The remainder of the paper proceeds as follows. Section 2 introduces the new framework, the new concept of constitutional stability and the random process for studying the convergence to constitutionally stable group structures. Section 3 provides conditions on constitutions and preferences for the existence of constitutionally stable group structures. Section 4 applies the general results to a model of job markets with labor unions. Finally, Section 5 concludes.

\section{The Model}

Let $N=\left\{i_{1}, \ldots, i_{n}\right\}$ be a finite set of players and let $M=\left\{c_{1}, \ldots, c_{m}\right\}$ be a finite set of groups.

Definition 1. A group structure $h$ is a mapping $h: M \longrightarrow 2^{N}$ assigning to each group $c \in M$ a subset of players $h(c) \in 2^{N}$, with $h(c)$ representing the members of group $c .^{9}$

A group structure $h$ indicates which players are members of which groups. For each $i \in N$, let $M_{h}(i)=\{c \in M \mid i \in h(c)\}$ be the set of groups player $i$ is a member of in the group structure $h$. The set of all group structures is denoted by $\mathcal{H}$, and the cardinality of $\mathcal{H}$ is $|\mathcal{H}|=2^{m n}$. A particular special case is the empty group structure $h^{\emptyset} \in \mathcal{H}$, with $h^{\emptyset}(c)=\emptyset$ for all $c \in M$. That is, no player is member of any group.

\footnotetext{
${ }^{9}$ Note that the tuple $(N, M, h)$ is simply a mathematical hypergraph. Therefore, from a technical point of view our definition of group structures also relates to the notions of conference structures (e.g., Myerson, 1980), many-to-many matchings (e.g., Roth, 1984) and social environments (e.g., Fershtman and Persitz, 2012).
} 
Example 1. Suppose there are three players and four groups, i.e., $N=\left\{i_{1}, i_{2}, i_{3}\right\}$ and $M=\left\{c_{1}, c_{2}, c_{3}, c_{4}\right\}$. Consider the case where all players are members of $c_{1}$, players $i_{2}$ and $i_{3}$ are also members of $c_{2}$ and $c_{3}$, while $c_{4}$ only contains $i_{1}$ as a member. This can be described formally by means of the following group structure $h$ (see also Figure 1):

$$
h(c)= \begin{cases}\left\{i_{1}, i_{2}, i_{3}\right\}, & \text { if } c=c_{1} \\ \left\{i_{2}, i_{3}\right\}, & \text { if } c \in\left\{c_{2}, c_{3}\right\} \\ \left\{i_{1}\right\}, & \text { if } c=c_{4} .\end{cases}
$$

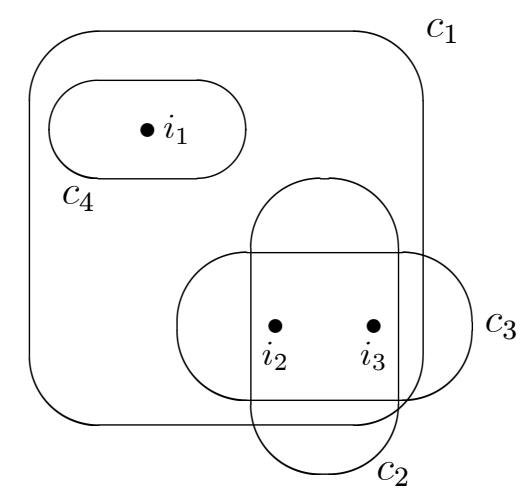

Figure 1: The group structure $h$.

\subsection{Constitutions}

Each $i \in N$ has rational preferences $\succeq^{i}$ over $\mathcal{H}$. The tuple $\succeq=\left(\succeq^{i}\right)_{i \in N}$ is called a preference profile. Given the preferences, players might have incentives to join or exit some group in a given group structure. We formally model the changes in the members of a given group by means of the symmetric difference \pm defined by $D^{\prime} \pm D=\left(D^{\prime} \backslash D\right) \cup\left(D \backslash D^{\prime}\right)$ for all $D^{\prime}, D \subseteq N$. Correspondingly, given a group $c \in M$ and a subset of players $D \subseteq N$, let $h \pm(c, D)$ be the group structure that is obtained from $h \in \mathcal{H}$ if the members of $c$ change due to the arrival and/or departure of the players in the deviating coalition $D$. More specifically, players in $D \cap h(c)$ leave the group and players in $D \backslash h(c)$ join it. ${ }^{10}$ Formally:

$$
(h \pm(c, D))\left(c^{\prime}\right):= \begin{cases}h(c) \pm D & \text { if } c=c^{\prime} \\ h\left(c^{\prime}\right) & \text { if } c \neq c^{\prime}\end{cases}
$$

\footnotetext{
${ }^{10}$ We use \pm instead of the usual symbol $\Delta$ for denoting the symmetric difference, in order to emphasize that it might be possible that at the same time new members enter a group while other members leave it.
} 
If $D \cap h(c)=\emptyset$, we just write $h+(c, D)$ instead of $h \pm(c, D)$ to stress the fact that no player leaves the group. If $D \subseteq h(c)$, we just write $h-(c, D)$ instead of $h \pm(c, D)$ to indicate that no player joins the group.

Given a group structure $h$ and a given group $c$, when a deviating coalition $D$ will change $h$ into $h \pm(c, D)$ ? The central assumption in our model is that each group could have different rules governing the composition of the group as well as the exit of already existing members and/or the arrival of new members. According to these rules, some deviations might be precluded, even if all deviating players would benefit from altering the group (i.e., even if $h \pm(c, D) \succeq^{i} h$ for all $\left.i \in D\right)$.

Some deviations might not be feasible, given the rules governing the composition of the group. For instance, some groups could have capacity constraints or some legal requirements regarding the type of member that could be part of the group. When this is the case, such groups could restrict the set of potential deviating coalitions that could be admitted into the group. Formally, for all $c \in M$ and $h \in \mathcal{H}$, we denote by $\mathcal{D}_{h}^{c} \subseteq 2^{N} \backslash\{\emptyset\}$ the set of feasible deviating coalitions.

Moreover, some feasible deviations could be blocked, given the rules governing the exit of existing members and/or the arrival of new members. According to these rules, each feasible deviating coalition $D \in \mathcal{D}_{h}^{c}$ would not be blocked only if there is a subgroup of existing members $S \in h(c)$ that agrees with the deviation. Formally, for each feasible deviating coalition $D \in \mathcal{D}_{h}^{c}$, the set of supporting coalitions that could grant its admission into the group $c$ is denoted by $S_{h}^{c}(D) \subseteq 2^{h(c)}$.

We introduce the notion of constitution to describe both the rules governing composition of the group and the conditions to fulfill in order to be accepted into the group.

Definition 2. The constitution $\mathcal{C}_{h}^{c}$ of group $c \in M$ in the group structure $h \in$ $\mathcal{H}$, is a pair $\mathcal{C}_{h}^{c}=\left(\mathcal{D}_{h}^{c}, S_{h}^{c}\right)$ where (i) $\mathcal{D}_{h}^{c} \subseteq 2^{N} \backslash\{\emptyset\}$ describes the set of feasible deviating coalitions, and (ii) for each $D \in \mathcal{D}_{h}^{c}, S_{h}^{c}(D) \subseteq 2^{h(c)}$ specifies a non-empty set of supporting coalitions that could grant the admission of the feasible deviating coalition into the group.

That is, for all $c \in M$ and $h \in \mathcal{H}$, the constitution of group $c$ in the group structure $h, \mathcal{C}_{h}^{c}$, consists of two components. The first one, $\mathcal{D}_{h}^{c}$, specifies which deviating coalitions are possible. Of course, it might be the case that in some group $c, \mathcal{D}_{h}^{c}=2^{N} \backslash\{\emptyset\}$ and then there are no restrictions on feasible deviations in such group. In other groups, however, certain changes in the composition of the group are not possible, and this is captured by $\mathcal{D}_{h}^{c} \subset 2^{N} \backslash\{\emptyset\}$. The second component of the constitution, $S_{h}^{c}(D)$, specifies for each feasible deviating coalition $D \in \mathcal{D}_{h}^{c}$ the supporting coalitions that could guarantee the admission of the deviating coalition into the group. Moreover, if $S \in \mathcal{S}_{h}^{c}(D) \backslash\{\emptyset\}$, we assume that $S^{\prime} \in \mathcal{S}_{h}^{c}(D)$ for all 
$S^{\prime} \supseteq S$. That is, if $S$ is a supporting coalition for a certain deviating coalition, all coalitions containing $S$ have also the power to support this deviation.

Let $\mathcal{C}^{c}=\left(\mathcal{C}_{h}^{c}\right)_{h \in \mathcal{H}}$ denote the constitutions of group $c$ in each possible group structure $h \in \mathcal{H}$. In the following, let $\mathcal{C}:=\left(\mathcal{C}^{c}\right)_{c \in M}$ denote the constitutions of each group in each possible group structure. The tuple $(N, M, \succeq, \mathcal{C})$ is called a society.

Example 2. Let $N=\left\{i_{1}, \ldots, i_{n}\right\}$ and $M=\left\{c_{1}, c_{2}, c_{3}\right\}$. As an example consider the following three specific constitutions:

(i) The constitution of group $c_{1}, \mathcal{C}^{c_{1}}$, is such that, $\mathcal{D}_{h}^{c_{1}}=\left\{D \subseteq N|| h\left(c_{1}\right) \pm D \mid \leq\right.$ $9, D \neq \emptyset\}$ and $\mathcal{S}_{h}^{c_{1}}(D)=\left\{S \subseteq h\left(c_{1}\right)|2 \cdot| S|>| h\left(c_{1}\right) \mid\right\}$ for all $h \in \mathcal{H}$ and $D \in \mathcal{D}_{h}^{c_{1}}$. According to the constitution, the number of members in this group cannot be larger than nine, and this restricts the set of feasible deviating coalitions. In order to be feasible, once the deviation takes place, the number of members cannot exceed nine. Moreover, decisions are taken by means of the majority rule. And then only the feasible deviating coalition that have the support of at least the majority of the existing members could be admitted into the group. ${ }^{11}$

(ii) The constitution of group $c_{2}, \mathcal{C}^{c_{2}}$, is such that, $\mathcal{D}_{h}^{c_{2}}=\left\{D \subseteq N \mid l \geq 3 \forall i_{l} \in\right.$ $D, D \neq \emptyset\}$ and $\mathcal{S}_{h}^{c_{2}}(D)=\left\{S \subseteq h\left(c_{2}\right) \mid h\left(c_{2}\right) \cap D \subseteq S\right\}$ for all $h \in \mathcal{H}$ and $D \in \mathcal{D}_{h}^{c_{2}}$. According to the constitution, deviations in this group require certain qualifications; that is, only certain type of individuals could be part of the group. In this specific example, players need an index of at least three. Moreover, none of the members has property rights granted by the constitution allowing them to veto the deviation of a feasible deviating coalition. That is, if a deviating coalition is feasible, the corresponding deviating players have the power to support themselves and then are free to enter or exit the group.

(iii) The constitution of group $c_{3}, \mathcal{C}^{c_{3}}$, is such that, $\mathcal{D}_{h}^{c_{3}}=2^{N} \backslash\{\emptyset\}$ and $\mathcal{S}_{h}^{c_{3}}(D)=$ $\left\{S \subseteq h\left(c_{3}\right) \mid i_{\bar{l}} \in S\right.$, where $\left.\bar{l} \geq l \forall i_{l} \in h\left(c_{3}\right)\right\}$ for all $h \in \mathcal{H}$ and $D \in \mathcal{D}_{h}^{c_{3}}$. According to the constitution, all potential deviating coalitions are feasible. However, the player with the highest index acts as a kind of dictator and has perfect property rights into the group. That is, she may decide about both: whether players may join the group as well as whether they may leave it. Every deviating coalition needs her approval in order to take place.

\footnotetext{
${ }^{11}$ These are the conditions to be satisfied by a deviating coalition in order to be feasible and having the support of the group. Of course, a feasible deviating coalititon with the support of the group will deviate to such group if she benefits from such deviation.
} 


\subsection{Stability}

A simple way to analyze the group structures that one might expect to emerge in the long run is to examine a sort of equilibrium requirement that no coalition benefits from altering the group structure. What about possible deviations? A group structure $h^{\prime}$ is obtainable from $h$ via the deviating coalition $D, D \subseteq N$, if (i) there is a unique group $c$ whose composition is changed; i.e., $h^{\prime}=h \pm(c, D)$, and (ii) the deviating coalition is feasible according to the constitution of group $c$; i.e., $D \in \mathcal{D}_{h}^{c}$. This definition identifies the possible resulting group structures once coalition $D$ has deviated from the existing group structure. The group structures that are obtainable from a given group structure $h$ are such that they only differ from $h$ in that a unique group has changed its composition via the deviation of a feasible deviating coalition according to the constitution of that group. ${ }^{12}$

Once identified all possible group structures that are obtainable from an existing group structure, different stability concepts could be studied. We propose the concept of constitutional stability to predict the group structures that are going to emerge in the long run in situations where the deviating coalition has to take into account the constitution of the group she want to deviate. A group structure $h$ is constitutionally stable if all coalitional deviations to some obtainable group structure are blocked.

Definition 3. Given the society $(N, M, \succeq, \mathcal{C})$, a group structure $h$ is constitutionally stable with respect to the constitutions $\mathcal{C}$ if for any $D \subseteq N, h \pm(c, D)$ obtainable from $h$ via $D$, we have that:

(i) $h \succeq^{i} h \pm(c, D)$ for at least one $i \in D \backslash h(c)$ or

(ii) in each supporting coalition $S \in \mathcal{S}_{h}^{c}(D)$ there exists $j \in S$ such that $h \succeq^{j}$ $h \pm(c, D)$.

Under the constitutions $\mathcal{C}$, a group structure $h \in \mathcal{H}$ is constitutionally stable if and only if any feasible deviation of some coalition $D \in \mathcal{D}_{h}^{c}$ to some obtainable group structure $h \pm(c, D)$ is deterred because at least one of the deviating players joining $c$ does not strictly benefit from deviating or at least one of the members of every supporting coalition $S \in \mathcal{S}_{h}^{c}(D)$ is not strictly better off from the deviation. ${ }^{13}$

\footnotetext{
${ }^{12} \mathrm{~A}$ more general definition of obtainable group structures could be obtained by allowing the deviating coalition to change the composition of several groups at the same time. However, since each group could have different constitutions imposing conflicting conditions for the deviating coalition to be feasible, we only allow the deviating coalition to change the composition of a unique group at a time. Of course, once a deviating coalition has changed a given group, other deviating coalition could change another group and so on.

${ }^{13}$ Assuming that players only deviate or support a deviation when their payoffs are strictly bigger has sense when transfers among players are not possible. This is in line with several other stability
} 
Therefore, we assume that moving from $h \in \mathcal{H}$ to $h \pm(c, D)$ does not necessarily need the consent of players leaving $c$. That is, some members of the group $c$ might have the power to force other members to leave $c$ even when the excluded players suffer from this exclusion. On the other hand, a player who is not in $c \in M$ cannot be forced to join $c$. Only if she strictly benefits, she will join it. Moreover, under the constitutions $\mathcal{C}$, the move from a group structure $h$ to any obtainable group structure $h \pm(c, D)$ needs the consent of every member of at least one of the supporting coalitions.

In the following, let $\mathcal{S T}(\mathcal{C})$ denote the set of constitutionally stable group structures with respect to the constitutions $\mathcal{C}$. Generically constitutionally stable group structures might fail to exist and this leads to the question of how the design of constitutions affects the (non-)existence of stable group structures. Moreover, even if constitutionally stable group structures exist, there is no guarantee that the society will reach one of them.

Let us formalize these ideas by adopting the notion of improving paths from Jackson and Watts $(2001,2002)$. An improving path is a sequence of group structures that can emerge when players join or leave some groups based on the improvement the resulting group structure offers them relative to the current one (see Jackson and Watts (2002), p.51). Each group structure in the sequence differs from the previous one in that one group is modified by a feasible deviating coalition and every player joining the group strictly prefers the resulting group structure to the current one. Moreover, the deviation should not be blocked and, hence, there should be a supporting coalition that strictly benefits from the deviation.

Given $h \in \mathcal{H}$ and $c \in M$, let $\mathcal{A}_{h}^{c}(\mathcal{C}):=\left\{D \in \mathcal{D}_{h}^{c} \mid \exists S \in \mathcal{S}_{h}^{c}(D)\right.$ such that $h \pm(c, D) \succ^{i} h \forall i \in S \cup(D \backslash h(c))$ with $\left.\emptyset \notin S \cup(D \backslash h(c))\right\}$ be the set of all feasible deviations that are not blocked. That is, the feasible deviations that strictly benefit all the deviating players joining the group and/or all members of at least one of the supporting coalitions. We restrict $\emptyset \notin S \cup(D \backslash h(c))$ in order to guarantee that there is at least one player that strictly benefits from deviating.

Definition 4. An improving path from $h_{0} \in \mathcal{H}$ to $h_{k} \in \mathcal{H}$ is a sequence of group structures $\left(h_{0}, h_{1}, \ldots, h_{k}\right)$ such that for all $0 \leq l<k$ we have $h_{l+1}=h_{l} \pm\left(c_{l}, D_{l}\right)$ with $c_{l} \in M$ and $D_{l} \in \mathcal{A}_{h_{l}}^{c_{l}}(\mathcal{C})$.

If there exists an improving path from $h \in \mathcal{H}$ to $h^{\prime} \in \mathcal{H}$, we write $h \mapsto h^{\prime}$. Moreover, let $I(h)=\left\{h^{\prime} \in \mathcal{H} \mid h \mapsto h^{\prime}\right\}$ be the set of group structures that can be concepts from literature, like strong stability of Dutta and Mutuswami (1997), pairwise stability of Sotomayor (1999), or core stability of Bogomolnaia and Jackson (2002) and Banerjee et al. (2001), for example. Notice that, as pointed out by one referee, the alternative assumption according to which a deviation is valid if some players are strictly better off and others are weakly better off, could lead to problems of equilibrium existence. 
reached by an improving path starting at $h$. Notice that $h$ is constitutionally stable if and only if there is no improving path starting at $h$; i.e., $I(h)=\emptyset$.

A set of group structures $H \subseteq \mathcal{H}$ is closed if there is no improving path leading out of it, i.e., $I(h) \subseteq H$ for all $h \in H$. Moreover, a set of group structures $H \subseteq \mathcal{H}$ with $|H| \geq 2$ is a cycle if for any pair $h, h^{\prime} \in H$, there exists an improving path connecting $h$ to $h^{\prime}$.

Lemma 1. Let the society $(N, M, \succeq, \mathcal{C})$ be given. There exists no closed cycle if and only if, for each group structure $h \in \mathcal{H}$ that is not constitutionally stable, there exists an improving path leading from this group structure to a constitutionally stable one.

Our Lemma 1 is a modification of Lemma 1 from Jackson and Watts (2002) and then we omit the proof. ${ }^{14}$ The non-existence of closed cycles not only implies existence of constitutionally stable group structures but it also guarantees that the society will reach one of these stable group structures. Consider the following random process which has been introduced for marriage problems by Roth and Vande Vate (1990). Start with an arbitrary group structure $h_{0} \in \mathcal{H}$. Each round $r \in \mathbb{N}_{\geq 0}$ a pair formed by a group and a deviating coalition $\left(c_{r}, D_{r}\right) \in M \times 2^{N}$ is drawn randomly with positive probability. If the deviating coalition is feasible and not blocked, i.e., $D_{r} \in \mathcal{A}_{h_{r}}^{c_{r}}(\mathcal{C})$, the process moves to $h_{r+1}:=h_{r} \pm\left(c_{r}, D_{r}\right)$. Otherwise it remains at $h_{r+1}:=h_{r}$.

Proposition 1. Let the society $(N, M, \succeq, \mathcal{C})$ be given. The random process described above always (i.e., for all $h_{0} \in \mathcal{H}$ ) converges with probability one to a constitutionally stable group structure if and only if there are no closed cycles.

In the context of one-to-one matching problems, the previous result has been established by Roth and Vande Vate (1990). The reasoning is the same and then we omit the proof. The intuition is straightforward. Since every feasible deviation is drawn with positive probability, every improving path has a positive probability. Therefore, if for every starting group structure there is an improving path leading to a constitutionally stable group structure, the random process converges to one of these stable structures for sure after finitely many steps.

In our formulation, the constitutions grant the group members a certain level of blocking power allowing them to inhibit changes in the composition of the group that do not conform to their own preferences. From the definition of constitutional stability we have that if the sets of feasible deviations and supporting coalitions shrink, the blocking power of each individual increases and the set of constitutionally

\footnotetext{
${ }^{14}$ Jackson and Watts (2002) show in slightly different terms that it is possible to find "pairwisestable" networks if there exists no closed cycle.
} 
stable group structures might become larger but never smaller. However, whether more blocking power really implies more stability, strongly depends on the adopted perspective of stability. Although the set of constitutionally stable group structures might become larger the greater the blocking power of the individuals, it could happen that the society will never reach one of these stable group structures because all improving paths leading to them could be destroyed and closed cycles could occur.

\section{Constitutional Rules and Preferences}

We now examine conditions under which the society will always induce a constitutionally stable group structure in the sense that, for every starting group structure, there is always an improving path leading to a constitutionally stable group structure. In order to get more intuition for this, let us consider a stylized example.

Example 3. Suppose there are three players $N=\left\{i_{1}, i_{2}, i_{3}\right\}$ and a unique group $M=\{c\}$. Let $h_{3}(c)=\left\{i_{3}\right\}, h_{5}(c)=\left\{i_{1}, i_{3}\right\}, h_{6}(c)=\left\{i_{2}, i_{3}\right\}$, and $h_{7}(c)=$ $\left\{i_{1}, i_{2}, i_{3}\right\}$. The corresponding feasible deviations are $D_{h_{3}}^{c}=D_{h_{5}}^{c}=D_{h_{6}}^{c}=D_{h_{7}}^{c}=$ $\left\{\left\{i_{1}\right\},\left\{i_{2}\right\},\left\{i_{3}\right\}\right\}$, while the supporting coalitions are given by $\mathcal{S}_{h_{l}}^{c}(D)=\{S \subseteq$ $\left.h_{l}(c) \mid i_{3} \in S\right\}$ for all $D \in \mathcal{D}_{h_{l}}^{c}$ where $l \in\{3,5,7\}$ and $\mathcal{S}_{h_{6}}^{c}(D)=\left\{S \subseteq h_{6}(c) \mid i_{2} \in S\right\}$ for all $D \in \mathcal{D}_{h_{6}}^{c}$. Moreover, the players' preferences are supposed to be as follows:

$$
\begin{aligned}
& h_{7} \succ^{i_{1}} h_{5} \succ^{i_{1}} h_{6} \sim^{i_{1}} h_{3} \succ^{i_{1}} \ldots \\
& h_{7} \succ^{i_{2}} h_{6} \succ^{i_{2}} h_{5} \sim^{i_{2}} h_{3} \succ^{i_{2}} \ldots \\
& h_{6} \succ^{i_{3}} h_{3} \succ^{i_{3}} h_{5} \succ^{i_{3}} h_{7} \succ^{i_{3}} \ldots
\end{aligned}
$$

It is not difficult to check that in this case the set $H=\left\{h_{3}, h_{6}, h_{7}, h_{5}\right\}$ forms a closed cycle because $\left(h_{3}, h_{6}, h_{7}, h_{5}, h_{3}\right)$ is an improving path (see Figure 2 ).

Inspecting this cycle in detail we can find a kind of irregularity in the constitutions: In $h_{3}, h_{5}$, and $h_{7}$, player $i_{3}$ is the only one who may decide about deviations and she even has the power to exclude the other players from the group. But after allowing $i_{2}$ to enter $c$ and moving to $h_{6}$, player $i_{3}$ looses her strong property rights and $i_{2}$ is able to grant $i_{1}$ access to the group. Moreover, not only do the constitutions exhibit a kind of irregularity but the players also disagree about the optimal form of the group. First, as mentioned before, $i_{3}$ can exclude $i_{1}$ or $i_{2}$ in $h_{7}$ against their will. If either this exclusion was not possible or the players agreed to being excluded and did not want to join the group again, the cycle would be splintered. Second, both players, $i_{2}$ and $i_{3}$, have the power to support a deviation of player $i_{1}$. The salient point is that both disagree about whether $i_{1}$ should be a member of the group or not. If there was a common agreement about this, one of the deviations would be blocked. 


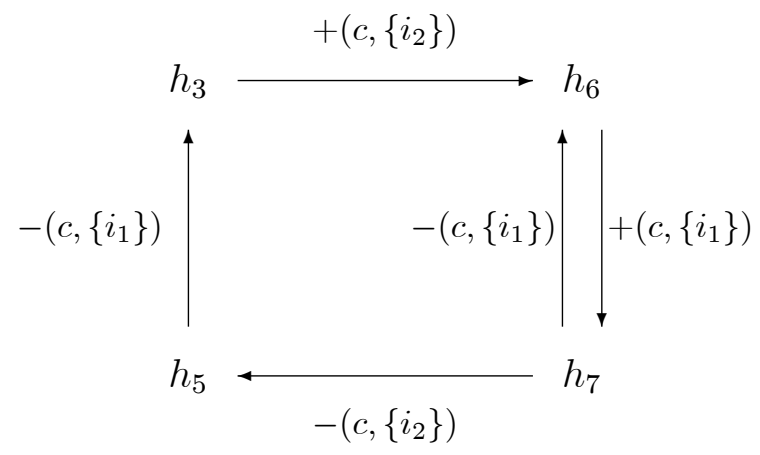

Figure 2: The cycle $H$.

As the example illustrates, in general there are three main factors which support the occurrence of closed cycles. First, constitutions might change strongly even if the group structure itself does not change much. Second, players might be forced to leave a group against their will. Third, there might be disagreement between the players who decide about the deviations. In order to guarantee the non-occurrence of closed cycles it is necessary to find reasonable restrictions on players' preferences and consistency conditions on the constitutions that allow us to control for all these factors.

Definition 5. Given a closed set $H \subseteq \mathcal{H}$, the constitutions $\mathcal{C}=\left(\mathcal{C}^{c}\right)_{c \in M}$ satisfy

- regularity with respect to $H$ if for all $h \in H$ and $c \in M$ we have:

(i) If $\bar{h}(c)=h(c) \cup \bar{D}$ for some $\bar{h} \in H$ and $\bar{D} \subseteq N \backslash h(c)$, then $\mathcal{D}_{h}^{c}=\mathcal{D}_{\bar{h}}^{c}$ and for all $D \in \mathcal{D}_{\bar{h}}^{c}$ and $\bar{S} \in \mathcal{S}_{\bar{h}}^{c}(D)$ there exists $S \in \mathcal{S}_{h}^{c}(D)$ with $S \subseteq \bar{S} \subseteq S \cup \bar{D}$.

(ii) If $D \in \mathcal{D}_{h}^{c}$ and $S \in \mathcal{S}_{h}^{c}(D)$ with $S \nsubseteq D$, then $h(c) \backslash(S \cup D) \notin \mathcal{S}_{h}^{c}(D)$.

- protection against eviction with respect to $H$ if for all $h \in H$ and $c \in M$ it holds $D \cap h(c) \subseteq S$ for all $D \in \mathcal{D}_{h}^{c}$ and $S \in \mathcal{S}_{h}^{c}(D)$;

- decomposability with respect to $H$ if for all $h \in H$ and $c \in M$, we have that $D \in \mathcal{D}_{h}^{c}$ implies $D^{\prime} \in \mathcal{D}_{h}^{c}$ and $\mathcal{S}_{h}^{c}(D)=\mathcal{S}_{h}^{c}\left(D^{\prime}\right)$ for all $D^{\prime} \subseteq D$.

The main motivation of regularity is to exclude the possibility of skipping back and forth between two group structures the whole time: Condition (i) states that the feasible deviations and corresponding supporting coalitions of each $c \in M$ may not vary extremely whenever $c$ changes. If further players are added to the group, the feasible deviations are supposed to remain the same and supporting coalitions change only as long as they might be complemented by new members. Thus, together with (ii) this implies that if a coalition $S \in \mathcal{S}_{h}^{c}(D)$ has the authority to support a deviation 
$D \in \mathcal{D}_{h}^{c}$, this cannot be reversed by another coalition which is neither associated to $S$ nor to $D$. If the constitutions satisfy protection against eviction, no player can be forced to leave a group $c \in M$ if she does not want to do it. Modifying $c$ always requires the consent of all deviating players (not only the consent of players who join the group). Decomposable constitutions exhibit a kind of independence property. If the deviation of a group of players is feasible, deviations of any subgroup of players are feasible as well and the corresponding supporting coalitions do not change.

Definition 6. A preference profile $\succeq$

- satisfies self-concern if $h \sim^{i} \bar{h}$ for all $i \in N$ and each pair of group structures $h, \bar{h} \in \mathcal{H}$ with $M_{h}(i)=M_{\bar{h}}(i)$ and $h(c)=\bar{h}(c)$ for all $c \in M_{h}(i)$.

- is lexicographic if each agent $i \in N$ has a preference ordering $\hat{\succeq}^{i}$ over $2^{M}$ such that $M_{h}(i) \grave{\succeq}^{i} M_{\bar{h}}(i)$ implies $h \succeq^{i} \bar{h}$ for all $h, \bar{h} \in \mathcal{H}$ with $M_{h}(i) \neq M_{\bar{h}}(i)$.

- is uniform if for all $i \in N, c \in M$, and $h, \bar{h} \in \mathcal{H}$ with $i \in h(c)=\bar{h}(c)$, $h-(c,\{k\}) \succ^{j} h$ implies $\bar{h}-(c,\{k\}) \succ^{i} \bar{h}$ and $h \succ^{j} h-(c,\{k\})$ implies $\bar{h} \succ^{i} \bar{h}-(c,\{k\})$ for $j \in h(c), k \in h(c) \backslash\{i, j\}$.

- is equable if for all $i \in N, c \in M$, and $h, \bar{h} \in \mathcal{H}$ with $i \in h(c)=\bar{h}(c)$, $h \succ^{j} h-(c,\{j\})$ for some $j \in h(c)$ implies $\bar{h} \succ^{i} \bar{h}-(c,\{i\})$ and $h-(c,\{j\}) \succ^{j} h$ for some $j \in h(c)$ implies $\bar{h}-(c,\{i\}) \succ^{i} \bar{h}$.

- is separable if for all $i \in N, c \in M$, and $h, \bar{h} \in \mathcal{H}$ with $i \in h(c) \subseteq \bar{h}(c)$ the two following conditions are satisfied:

(i) $\bar{h}-(c, D) \succ^{i} \bar{h}$ if and only if $h-(c, D) \succ^{i} h$ for all $\emptyset \neq D \subseteq h(c) \backslash\{i\}$.

(ii) $\bar{h}+(c, D) \succ^{i} \bar{h}$ if and only if $h+(c, D) \succ^{i} h$ for all $\emptyset \neq D \subseteq N \backslash \bar{h}(c)$.

Self-concern is a kind of independence property. Player $i$ neither benefits nor suffers if the group structure changes in such a way that $i$ is not affected directly. The definition of lexicographic preferences is adapted from Dutta and Masso (1997). Under this requirement, player $i$ is mainly concerned about the groups themselves where she is a member of and less about who the other members are. Only if $M_{h}(i)=$ $M_{\bar{h}}(i)$, might she care about the other players in her groups. If the preferences of the players are uniform and a player leaves a group, either all remaining members benefit from this deviation or none of them. Note that this is supposed to be independent of the form the other groups have. Under equability player $i$ wants to stay in a group $c \in M$ only if the other members also want to stay. Suppose, for example, the groups generate a payoff which is distributed equally among the members. Then, if another 
player has an incentive to leave $c$, the same goes for $i$. Separability as introduced here is a variation of the same-named concept from Banerjee et al. (2001). The idea is that player $i$ 's support for a certain leaving or joining group $D$ is independent of the form the group actually has.

Combining these restrictions we can formulate new results regarding requirements on constitutions and preferences which guarantee the non-existence of (closed) cycles and the convergence to a constitutionally stable group structure. ${ }^{15}$

Proposition 2. Let a society $(N, M, \succeq, \mathcal{C})$ be given where all constitutions satisfy protection against eviction with respect to a closed set $H \subseteq \mathcal{H}$. If the players' preferences satisfy equability and self-concern, there exist no cycles in $H$.

All the proofs can be found in the Appendix. The requirements of Proposition 2 control the three factors identified above which might cause the occurrence of closed cycles. Equability and self-concern, for example, impose restrictions on the players' preferences. Both conditions together guarantee that there is only little disagreement about the optimal form of each group $c \in M$. Moreover, protection against eviction with respect to $H$ has two consequences. On the one hand, as the definition directly implies, players cannot be forced to leave a group if they do not agree to this. On the other hand, it also indirectly ensures that the constitutions do not change too strongly whenever a feasible deviation takes place. More specifically, $S \in \mathcal{S}_{h}^{c}(D)$ implies $h(c) \backslash S \notin \mathcal{S}_{h}^{c}(D)$ for all $h \in H, c \in M$, and $D \in \mathcal{D}_{h}^{c}$. The interpretation is similar to regularity. If $S$ has the power to support a deviation of $D$, this cannot be reversed by other supporting coalitions.

Proposition 3. Let a society $(N, M, \succeq, \mathcal{C})$ be given where all constitutions satisfy protection against eviction with respect to a closed set $H \subseteq \mathcal{H}$. If the players' preferences are lexicographic, there exist no cycles in $H$.

The intuition behind Proposition 3 is similar to the one of Proposition 2. Obviously, the only difference is that the preferences are not supposed to satisfy equability and self-concern but here they are lexicographic. Therefore, even if there is some disagreement about the optimal form of the groups, it is relegated to a secondary role.

Propositions 2 and 3 exclude the existence of closed cycles by imposing protection against eviction. Indeed, it is not possible to drop or to relax this assumption without reinforcing the requirements on players' preferences if one wants to avoid closed cycles.

\footnotetext{
${ }^{15} \mathrm{An}$ alternative way to exclude the occurrence of closed cycles is to look for constitutions that allow for a common ranking (cf. Banerjee et al., 2001; Farrell and Scotchmer, 1988). See the Appendix.
} 
Proposition 4. Let a society $(N, M, \succeq, \mathcal{C})$ be given. Assume all constitutions are decomposable and regular with respect to a closed set $H \subseteq \mathcal{H}$. Moreover, suppose the players' preferences satisfy self-concern and are separable, uniform and equable. Then, there exist no closed cycles in $H$.

As the definition directly implies, regularity inhibits the constitutions from varying too extremely and, similar to Proposition 2, equability and self-concern guarantee a certain degree of consent about the optimal form of the group structure. In addition to this, due to separability and uniformity, in most situations the players are not forced to leave their groups if they do not agree to this. If, for example, some player's entry is supported by a certain coalition, the corresponding members will not change their minds, even if the group is altered strongly. Thus, the player will only leave again the group if she benefits from leaving it.

As before, it is possible to replace equability and self-concern in Proposition 4 by lexicography. The intuition is the same: The optimal form of the groups is relegated to a secondary role.

Proposition 5. Let the society $(N, M, \succeq, \mathcal{C})$ be given. Assume all constitutions are decomposable and regular with respect to a closed set $H \subseteq \mathcal{H}$. Moreover, suppose the preferences of the players are separable, uniform and lexicographic. Then, there exist no closed cycles in $H$.

In the next section, we show how these results that explore different constitutional arrangements and individuals' preferences guaranteeing stability could be applied to a particular application of our general framework, namely to job markets with labor unions.

\section{Many-to-many Matching Markets}

One of the most interesting features of our model is its versatile applicability since overlapping group structures appear in many environments. Consider, for example, many-to-many matching markets. The main primitives of these markets are two finite sets of players $E$ and $F$, where the members of $E$ are usually interpreted as employees (or workers) and the members of $F$ as firms (see, e.g., Roth, 1984). A (two-sided) many-to-many matching $\mu \subseteq E \times F$ is then simply a collection of worker-firm pairs indicating which employees are working for which firms. Both sides of the market are supposed to have preferences over all possible matchings. Thereby, the employees are classically assumed to care only about which firms they work for but not about who their co-workers might be. The owners, on the other hand, are only concerned about the employees working for their firm: "This involves 
an assumption that workers are indifferent to who their co-workers might be, and firms are indifferent to whether their employees moonlight at other jobs." (Roth, 1984, P. 51)

Many-to-many matching markets can be embedded into our setting in a straightforward way. Let each $c \in M$ represent a firm, i.e., $M:=F$. Since in our model the groups do not act as players, we suppose that each firm $c \in M$ has exactly one owner $o_{c} \in O$. That is, we assume that the set of players $N:=E \cup O$ can be decomposed into two (disjoint) subsets, the employees $E$ and the owners $O$. Given these preliminaries, each matching $\mu \subseteq E \times F$ can be represented by the firm structure $h^{\mu} \in H$ which is defined via $h^{\mu}(c)=\{i \in E \mid(i, c) \in \mu\} \cup\left\{o_{c}\right\}$ for all $c \in M=F$. In order to be in line with the classical literature on many-to-many matchings, we assume that each owner has no incentive for leaving her firm or for joining any other firm, i.e., we are only interested in the case $O \cap h(c)=\left\{o_{c}\right\}$ for $h \in \mathcal{H}$ and $c \in M$. Nevertheless, since we do not exclude certain firm structures a priory, for technical reasons, we have to define preferences over firm structures where this requirement is not met. Roth's assumptions on players' preferences imply that each employee $i \in E$ is indifferent among all firm structures where she is working for the same set of firms, i.e., $h \sim^{i} \bar{h}$ for all $h, \bar{h} \in \mathcal{H}$ with $M_{h}(i)=M_{\bar{h}}(i)$. Moreover, given $c \in M$ and $O \cap h(c)=\left\{o_{c}\right\}$, the assumptions imply $h \sim^{o_{c}} \bar{h}$ whenever $h(c)=\bar{h}(c)$. For the (pathological) case where $O \cap h(c) \neq\left\{o_{c}\right\}$, we assume $h \pm\left((O \cap h(c)) \pm\left\{o_{c}\right\}\right) \succ^{o_{c}} h$. Therefore, the preferences of all employees are lexicographic. And, restricted to the set $H:=\left\{h \in \mathcal{H} \mid O \cap h(c)=\left\{o_{c}\right\} \forall c \in M\right\}$, the same holds for the owners, too.

Our formalization of constitutions allows us studying different levels of authority of the owners in a flexible way. For instance, in many countries (especially in Europe) employees are organized in labor unions representing the interests of their members. These unions may guarantee a quite strong protection against dismissal to the workers and, in the short run, the consent of a worker is needed if the owner wants her to leave the firm. Many-to-many matching theory, however, usually concentrates on job markets without strong protection against dismissal like the US job market, for example, and neglects the impact of labor unions. Due to its versatility our model provides an appropriate framework for examining and comparing these different job markets in a convenient way. The remainder of this section is devoted to studying the existence of constitutionally stable firm structures in three environments that differ in the level of authority that the owners and the workers could have. The three scenarios analyzed are denoted Protection against Dismissal; Hire and Fire; and Slavery. In the three scenarios, the owner is the only one who may decide about hiring new workers. The following table shows the differences among the three scenarios. 


\begin{tabular}{|l|c|c|}
\hline $\begin{array}{c}\text { Protection against Dismissal } \\
\text { The consent of a worker } \\
\text { is needed if the owner } \\
\text { wants her to leave the firm }\end{array}$ & $\begin{array}{c}\text { Hire and Fire } \\
\text { power to fire her } \\
\text { employees even if those } \\
\text { do not want to leave }\end{array}$ & $\begin{array}{c}\text { The owner has the } \\
\text { power to fire her } \\
\text { employees even if those } \\
\text { do not want to leave }\end{array}$ \\
$\begin{array}{c}\text { The employees may } \\
\text { always leave the firms }\end{array}$ & $\begin{array}{c}\text { The owner has the } \\
\text { always leave the firms }\end{array}$ & $\begin{array}{c}\text { power to decide } \\
\text { whether the employees } \\
\text { may leave the firm or not }\end{array}$ \\
\hline
\end{tabular}

Moving from Protection against Dismissal to Hire and Fire, and from Hire and Fire to Slavery, increases more and more the degree of authority of the owners while decreasing the one of the workers. Roth (1984) shows that there are firmoptimal and worker-optimal stable outcomes in a general, completely symmetric model, in which firms may employ any number of workers (like in the model of Kelso and Crawford, 1982), workers may take more than one job (while Kelso and Crawford (1982) impose that workers may work for only one firm), and each worker has preferences over sets of jobs. Roth (1984) also shows that the stable outcome which is best for all the firms it is the worst stable outcome for all the workers, and vice-versa. These results of Roth (1984) raise questions about the nature and underlying causes of such polarization of interests. In the following, we will study the relationship between the degree of authority of each side of the market, the existence of stable firm structures and the persistence of Roth's "polarization of interests".

Following Roth (1984), we assume that the employees are allowed to accept as many jobs as they want to. Moreover, the firms have unlimited capacity to hire workers, i.e., given $O \cap h(c)=\left\{o_{c}\right\}$ for $h \in \mathcal{H}$ and all $c \in M$, every possible deviation of the employees is feasible. For sake of completeness, we also have to consider the case where an owner is not part of her firm or other owners are contained in it. Then, we assume that the only feasible deviation in this case is to add the owner and to delete all other owners. Formally,

$$
\mathcal{D}_{h}^{c}= \begin{cases}2^{E}, & \text { if } O \cap h(c)=\left\{o_{c}\right\} \\ (O \cap h(c)) \pm\left\{o_{c}\right\}, & \text { if } O \cap h(c) \neq\left\{o_{c}\right\}\end{cases}
$$

4.1. Protection against Dismissal. First we consider the case where unions may guarantee a quite strong protection against dismissal to the workers and the owners do not have the authority to fire them. Each employee is always free to terminate her job if she has an incentive to do it. These considerations lead to the following 
set of supporting coalitions:

$\mathcal{S}_{h}^{c}(D)= \begin{cases}\left\{S \subseteq h(c) \mid D \cap h(c) \subseteq S \text { and } o_{c} \in S\right\}, & \text { if } O \cap h(c)=\left\{o_{c}\right\} \text { and } D \nsubseteq h(c) \\ \{S \subseteq h(c) \mid D \subseteq S\}, & \text { if } O \cap h(c)=\left\{o_{c}\right\} \text { and } D \subseteq h(c) \\ \{\emptyset\}, & \text { if } O \cap h(c) \neq\left\{o_{c}\right\}\end{cases}$

In case of $O \cap h(c) \neq\left\{o_{c}\right\}$, we assume that the empty set is the only supporting coalition and, thus, these firm structures are not stable by construction. From Proposition 3 we get the following corollary:

Corollary 1. There are no cycles in "Protection against Dismissal".

At first sight, this result might be slightly surprising because in many studies about two-sided many-to-many matchings the existence of stable matchings is an issue (e.g., Sotomayor, 2004). This is mainly due to the fact that this literature examines environments where the owners are free to fire a worker if they benefit from it. Indeed, protection against dismissal is the driving force of Corollary 1. Let $\mathcal{S T}^{\mathrm{PD}}$ denote the set of stable firm structures in Protection against Dismissal. This set contains the worker-optimal firm structures which are defined as follows. Suppose $\bar{M}^{i} \subseteq M$ is a set of firms which is mostly preferred by player $i \in E$. Then, if $h^{w o}$ is given by $h^{w o}(c)=\left\{i \in E \mid c \in \bar{M}^{i}\right\} \cup\left\{o_{c}\right\}$ for all $c \in M$, every employee is assigned to a set of firms she preferably wants to work for and, thus, she obviously has no incentive for deviating.

4.2. Hire and Fire. We now focus on job markets without strong protection against dismissal. Translated to the model considered here, this means that the owners have the right to fire workers even if these do not want to leave the firms. This aspect can be captured by considering the following supporting coalitions:

$$
\mathcal{S}_{h}^{c}(D)= \begin{cases}\left\{S \subseteq h(c) \mid o_{c} \in S\right\}, & \text { if } O \cap h(c)=\left\{o_{c}\right\}, D \nsubseteq h(c) \\ \left\{S \subseteq h(c) \mid D \subseteq S \text { or } o_{c} \in S\right\}, & \text { if } O \cap h(c)=\left\{o_{c}\right\}, D \subseteq h(c) \\ \{\emptyset\}, & \text { if } O \cap h(c) \neq\left\{o_{c}\right\}\end{cases}
$$

Let $\mathcal{S} \mathcal{T}^{\mathrm{HF}}$ be the set of stable firm structures in "Hire and Fire". Note that $\mathcal{S} \mathcal{T}^{\mathrm{HF}} \subseteq \mathcal{S} \mathcal{T}^{\mathrm{PD}}$. However, it is well known that without further assumptions the existence of stable firm structures in Hire and Fire is not guaranteed. Thus, in order to exclude existence of closed cycles it is necessary to impose further restrictions on constitutions or preferences. For instance, we could proceed similarly as in Proposition 5 since the preferences of the employees are lexicographic and the constitutions in Hire and Fire are not only decomposable but also regular with respect to $H$. However, since the owners are the only players who have decision making 
power and they never leave their firm, uniformity is not needed and it is sufficient to additionally assume that the owners' preferences are separable.

Proposition 6. If the preferences of the owners are separable, there exists no closed cycle in Hire and Fire.

Similar to our result, Kojima and Unver (2008) have shown in the context of two-sided many-to-many matchings that if workers and owners have, respectively, "substitutable" (see Roth, 1984) and "responsive" (see Roth, 1985) preferences, then there always exists a sequence of pairwise and individual deviations leading to a pairwise stable matching. Notice that, in our model, we fix $c$ in order to determine the possible deviations and other $c$ 's are not affected when a group of players deviates from $c$. So, we cannot handle pairwise deviations affecting simultaneously several connections. However, we can model individual deviations. Notice that responsiveness of the owners' preferences implies separability which in turn implies substitutability (converse implications do not hold) if only individual deviations are feasible. Thus, our assumptions in Proposition 6 are less restrictive than the ones imposed by Kojima and Unver (2008) for the case where only individual deviations are feasible. Therefore, Proposition 6 provides alternative conditions on the preferences of the owners to guarantee that there always exists a sequence of individual deviations leading to an individually stable matching.

4.3. Slavery. Roughly speaking, "Slavery" is the counter-position of Protection against Dismissal. Here, the owners not only have the power to decide about new employees but also about whether workers may leave their firm or not:

$$
\mathcal{S}_{h}^{c}(D)= \begin{cases}\left\{S \subseteq h(c) \mid o_{c} \in S\right\}, & \text { if } O \cap h(c)=\left\{o_{c}\right\} \text { and } D \nsubseteq h(c) \\ \left\{S \subseteq h(c) \mid o_{c} \in S\right\}, & \text { if } O \cap h(c)=\left\{o_{c}\right\} \text { and } D \subseteq h(c) \\ \{\emptyset\}, & \text { if } O \cap h(c) \neq\left\{o_{c}\right\}\end{cases}
$$

From Proposition 3 we get the following corollary:

Corollary 2. Every improving path in "Slavery" leads to a constitutionally stable firm structure.

Let $\mathcal{S} \mathcal{T}^{\text {SL }}$ denote the set of stable firm structures in "Slavery". Analogously to worker-optimal firm structures it is possible to define firm-optimal firm structures. Let $\hat{E}^{c} \subseteq E$ be a set of employees which is mostly preferred by player $o_{c}$ and define $h^{f o}$ by $h^{f o}(c)=\hat{E}^{c} \cup\left\{o_{c}\right\}$ for all $c \in M$. Then, none of the owners has an incentive for deviating and, thus, the firm structure is stable in Slavery. Note that $\mathcal{S T}^{\mathrm{HF}} \subseteq \mathcal{S} \mathcal{T}^{\mathrm{SL}}$. Hence, a firm structure is stable in Hire and Fire if and only if it is stable in Protection against Dismissal and Slavery, i.e., $\mathcal{S} \mathcal{T}^{\mathrm{HF}}=\mathcal{S} \mathcal{T}^{\mathrm{PD}} \cap \mathcal{S} \mathcal{T}^{\mathrm{SL}}$. According 
to Corollary 1 and Corollary 2 there are no cycles in Protection against Dismissal and Slavery. Therefore, a simple algorithm for finding stable firm structures in Hire and Fire (in case they exist) is to determine the sets of constitutionally stable firm structures in the other two settings and then to check whether the intersection of these sets is non-empty.

4.4. Roth's Polarization of Interests. Although we have $\mathcal{S} \mathcal{T}^{\mathrm{HF}} \subseteq \mathcal{S} \mathcal{T}^{\mathrm{PD}}$, the converse inclusion does not necessarily hold. Therefore, there might exist firm structures which are stable in Protection against Dismissal that would not be stable if the owners' level of authority is sufficiently high. In particular, due to the characteristics of Hire and Fire, if $h \in \mathcal{S} \mathcal{T}^{\mathrm{PD}}$ but $h \notin \mathcal{S} \mathcal{T}^{\mathrm{HF}}$, there is at least one owner who would like to fire some of her employees against their will. This already indicates that the interests of both sides of the market might be opposed in a way. For deepening these considerations further we need to enhance separability.

Definition 7. A preference profile $\succeq$ is strongly separable if for all $i \in N, c \in M$, and $h, \bar{h} \in \mathcal{H}$ with $i \in h(c) \subseteq \bar{h}(c)$, the two following conditions are satisfied:

(i) $\bar{h}-(c, D) \succ^{i} \bar{h}$ if and only if $h-(c, D) \succ^{i} h$ for all $\emptyset \neq D \subseteq h(c)$.

(ii) $\bar{h}+(c, D) \succ^{i} \bar{h}$ if and only if $h+(c, D) \succ^{i} h$ for all $\emptyset \neq D \subseteq N \backslash \bar{h}(c)$.

Strong separability is a stronger requirement than separability. Under strong separability, player $i$ 's support for a certain leaving or joining coalition is independent of the other members of the connection even if $i$ belongs to the deviating coalition and leaves the group. Translated to Hire and Fire, this basically means that $i$ 's preference about whether to work for a firm $c \in M$ or not is independent of the other firms she is working for.

Proposition 7. Assume the workers' preferences are strongly separable and the owners' preferences are separable. Moreover, suppose the worker-optimal firm structure $h^{w o}$ is uniquely determined. Then, $h^{w o} \in \mathcal{S} \mathcal{T}^{H F}$ if and only if $\mathcal{S} \mathcal{T}^{P D}=\mathcal{S} \mathcal{T}^{H F}$.

Proposition 7 is in line with Roth (1984). Under the requirement that the preferences of the owners and employees are substitutable, the author finds a "conflict of interest between agents on opposite sides [of the market]" (Roth (1984), p.47). A similar conflict also arises here: Given (strong) separability of the players' preferences, the stable outcome which would be blocked first by the owners is the worker-optimal firm structure.

Notice that $\mathcal{S T}^{\mathrm{HF}} \subseteq \mathcal{S} \mathcal{T}^{\mathrm{SL}}$, while the converse inclusion does not necessarily hold. Therefore, there might exist firm structures which are stable in Slavery that would not be stable if the owners' level of authority is lower. In particular, due to 
the characteristics of Hire and Fire, if $h \in \mathcal{S} \mathcal{T}^{\mathrm{SL}}$ but $h \notin \mathcal{S} \mathcal{T}^{\mathrm{HF}}$, there is at least one worker who would like to leave some firm and this deviation is blocked by the owner of such firm.

Proposition 8. Assume the workers' preferences are strongly separable and the owners' preferences are separable. Moreover, suppose the firm-optimal firm structure $h^{\text {fo }}$ is uniquely determined. Then, $h^{\text {fo }} \in \mathcal{S} \mathcal{T}^{H F}$ if and only if $\mathcal{S} \mathcal{T}^{S L}=\mathcal{S} \mathcal{T}^{H F}$.

Proposition 8 has two implications. First, it shows that the owners can enforce the firm structure which is most beneficial for them if they have a high level of authority. Second, this firm structure would be the first firm structure which is rejected by the employees. Given (strong) separability of the players' preferences, both the worker-optimal firm structure and the firm-optimal firm structure are stable in Hire and Fire. Notice that we abstract from factors like wages or the working environment in our model and this implies that Roth's "polarization of interests" seems to achieve great generality. In fact, the result of Proposition 8 extends and reinforces the interpretation of Proposition 7 in a straightforward way: Starting from Hire and Fire, each side of the market would be worse off if the other side obtains more degree of authority (in case the (strong) separability condition of the players' preferences does not hold). If labor unions narrow the owners' level of authority (moving from Hire and Fire to Protection against Dismissal), the employees would benefit from this reduction of the degree of authority of the owners by stabilizing the work-optimal firm structure. And vice versa, an increase in the degree of authority of the owners (moving from Hire and Fire to Slavery) would stabilize the firm-optimal firm structure benefiting the owners.

\section{Conclusion}

We have developed a general theoretical framework in order to study the stability of any overlapping coalition setting, and not only of specific cooperative or noncooperative games with overlapping coalitions. We have introduced the notion of constitution in order to model for each group the rules governing both the composition of the group and the conditions needed to leave the group and/or to become a new member of the group. The constitutions are flexible enough to capture a wide spectrum of possible applications. We have then proposed the concept of constitutional stability to study the group structures that are going to emerge at equilibrium in overlapping coalition settings. This concept generalizes previous stability concepts in the literature in which the constitutional rules were exogenously given or not explicitly considered. 
We have examined both the existence of constitutionally stable group structures as well as whether the society will reach one of these stable group structures. We have shown that the society induces a constitutionally stable group structure if and only if the constitutions inhibit the occurrence of closed cycles. We have provided requirements on constitutions and individuals' preferences guaranteeing that, from every initial group structure, there always exists an improving path leading to a constitutionally stable group structure. Furthermore, by embedding many-to-many matchings into our setting, we have shown how these results could be useful to identify the constitutionally stable group structures in this particular environment. We have shown that the job market becomes stable if the degree of authority of one side of the market becomes sufficiently high. We have found a variation of Roth's "polarization of interests" (cf. Roth, 1984) between employers and employees: Each side of the market would be worse off if the other side obtains more degree of authority.

Some extensions of our model are left for future work. First, it might be worth analyzing which results could be obtained if players act farsightedly. ${ }^{16}$ Take a group structure and assume that there is a single profitable myopic deviation that results in an unstable group structure and that there is a single further myopic deviation that results in a stable group structure. Suppose those in the initial supporting coalition are made worse off by this sequence of changes. The initial group structure would not then be considered stable by the current definition, but perhaps should be if players are not myopic but farsighted and join or leave groups based on the improvement the end group structure offers relative to the current one. Another natural extension is to examine situations where it is possible to add new players or groups to the society. To incorporate this kind of dynamics, it would be necessary to relax the assumption of fixed sets of players and groups. Finally, it might be worth analyzing other specific environments, like research collaborations and social clubs, where individuals are allowed to engage in several groups at the same time.

\section{Acknowledgments}

We thank the editor and two anonymous referees for their valuable comments and suggestions to improve the paper. Ana Mauleon and Vincent Vannetelbosch are Senior Research Associates of the National Fund for Scientific Research (FNRS). Financial support from Spanish Ministry of Economy and Competition, FEDER

\footnotetext{
${ }^{16}$ In the context of coalition or network formation several stability concepts for farsighted agents that care about the end consequences of their acts have been proposed (see, e.g., Herings et al., 2009; Page and Wooders, 2009; Page et al., 2005).
} 
and European Union under the project ECO2015-64467-R (MINECO/FEDER, UE), and the Belgian French speaking community ARC project Nr. 15/20-072 of the Université Saint-Louis - Bruxelles is gratefully acknowledged. Nils Roehl gratefully acknowledges financial support by the German Research Foundation (DFG) within the Collaborative Research Center "On-The-Fly Computing" (SFB 901).

\section{Appendix}

\section{A.1. Proofs.}

Proof of Proposition 2. Let $\left(h_{0}, \ldots, h_{k}\right)$ with $h_{0}, \ldots, h_{k} \in H$ be an improving path. Moreover, suppose $h_{0}=h_{k}$, that is, suppose $\left\{h_{0}, \ldots, h_{k}\right\}$ forms a cycle. By construction of improving paths there exists $c_{0} \in M$ and $D_{0} \in \mathcal{D}_{h_{0}}^{c_{0}}$ with $h_{1}=$ $h_{0} \pm\left(c_{0}, D_{0}\right)$.

Case 1: $D_{0} \nsubseteq h_{0}\left(c_{0}\right)$, i.e., there exists $i_{0} \in D_{0} \backslash h_{0}\left(c_{0}\right)$.

Thus, $h_{1} \succ^{i_{0}} h_{0}$. Because all players are self-concerned this implies

$$
h_{1} \succ^{i_{0}} h_{0} \sim^{i_{0}} h_{0} \pm\left(c_{0}, D_{0} \backslash\left\{i_{0}\right\}\right)=h_{1}-\left(c_{0},\left\{i_{0}\right\}\right) .
$$

In other words, after joining the group player $i_{0}$ has no incentive to leave it unilaterally. By equability this is true for all other $i \in h_{1}\left(c_{0}\right)$. Moreover, let $D \in \mathcal{D}_{h_{1}}^{c_{0}}$ with $D \cap h_{1}\left(c_{0}\right) \neq \emptyset$ and let $i \in D \cap h_{1}\left(c_{0}\right)$. Then:

$$
h_{1} \succ^{i} h_{1}-\left(c_{0},\{i\}\right) \sim^{i}\left(h_{1}-\left(c_{0},\{i\}\right)\right) \pm\left(c_{0}, D \backslash\left\{i_{0}\right\}\right)=h_{1} \pm\left(c_{0}, D\right) .
$$

Because the constitutions satisfy protection against eviction by assumption, no player can be forced to leave a group against her will. Thus, all players in $h_{1}\left(c_{0}\right) \cap D$ would block the deviation from $h_{1}$ to $h_{1} \pm\left(c_{0}, D\right)$. We will show next that the same is also true in $h_{2}$. To this end, let $c_{1} \in M$ and $D_{1} \in \mathcal{D}_{h_{1}}^{c_{1}}$ with $h_{2}=h_{1} \pm\left(c_{1}, D_{1}\right)$. If $c_{1}=c_{0}$, the previous discussion implies $D_{1} \cap h_{1}\left(c_{0}\right)=\emptyset$ and, by similar arguments as before, it can be shown that $h_{2} \succ^{i} h_{2} \pm\left(c_{0}, D\right)$ for all $i \in h_{2}\left(c_{0}\right)$ and $D \in \mathcal{D}_{h_{2}}^{c_{0}}$ with $i \in D$. However, if $c_{1} \neq c_{0}$, then $h_{2}\left(c_{0}\right)=h_{1}\left(c_{0}\right)$. Thus, by equability $h_{2} \succ^{i} h_{2} \pm\left(c_{0}, D\right)$ for all $i \in h_{2}\left(c_{0}\right)$ and $D \in \mathcal{D}_{h_{2}}^{c_{0}}$ with $i \in D$. Iterating these arguments implies $h_{l} \succ^{i} h_{l}-\left(c_{0}, D\right)$ for all $1 \leq l \leq k, i \in h_{l}\left(c_{0}\right)$ and $D \in \mathcal{D}_{h_{l}}^{c_{0}}$ with $i \in D$. In particular, if $h_{0}=h_{k}$, then $h_{0}=h_{k} \succ^{i_{0}} h_{k}-\left(c_{0}, D_{0}\right)=h_{1}$ and, thus, $i_{0}$ would have blocked deviating to the group structure $h_{1}$.

Case 2: $D_{0} \subseteq h_{0}\left(c_{0}\right)$, i.e., $h_{1}=h_{0}-\left(c_{0}, D_{0}\right)$.

Thus, $h_{1}\left(c_{0}\right) \subsetneq h_{0}\left(c_{0}\right)$ and, moreover, $h_{0}-\left(c_{0}, D_{0}\right) \succ^{i_{0}} h_{0}$ by protection against eviction. Let $i_{0} \in D_{0}$. Because $h_{0}=h_{k}$, there must be $1 \leq k^{\prime} \leq k-1$ and $D \in \mathcal{D}_{h_{k^{\prime}}}^{c_{0}}$ with $h_{k^{\prime}+1}=h_{k^{\prime}} \pm\left(c_{0}, D\right)$ and $i_{0} \in D$. In particular, $h_{k^{\prime}+1} \succ^{i_{0}} h_{k^{\prime}}$. Similar to Case 1, exploiting that all players are self-concerned yields

$$
h_{k^{\prime}+1} \succ^{i_{0}} h_{k^{\prime}} \sim^{i_{0}} h_{k^{\prime}} \pm\left(c_{0}, D \backslash\left\{i_{0}\right\}\right)=h_{k^{\prime}}-\left(c_{0},\left\{i_{0}\right\}\right) .
$$


Therefore, equability implies $h_{k^{\prime}+1} \succ^{i} h_{k^{\prime}+1}-\left(c_{0},\{i\}\right)$ for all $i \in h_{k^{\prime}+1}\left(c_{0}\right)$. Now, by advancing analog arguments as in Case 1 it is possible to show that this also yields $h_{l} \succ^{i} h_{l}-\left(c_{0}, D\right)$ for all $k^{\prime}+1 \leq l \leq k, i \in h_{l}\left(c_{0}\right)$ and $D \in \mathcal{D}_{h_{l}}^{c_{0}}$ with $i \in D$. In particular, this is also true for $h_{0}=h_{k}$. But this contradicts again $h_{0}-\left(c_{0}, D_{0}\right)=h_{1} \succ^{i_{0}} h_{0}$.

Proof of Proposition 3. Let $\left(h_{0}, h_{1}, \ldots, h_{k}\right)$ be an improving path in $H$. We will show by induction that there is always at least one player $i \in N$ with $M_{h_{k}}(i) \neq$ $M_{h_{0}}(i)$ and $h_{k} \succ^{i} h_{0}$. Thus, $h_{k} \neq h_{0}$.

$k=1$ : According to the definition of an improving path and because all constitutions satisfy protection against eviction, at least one of the deviating players strictly benefits from moving to $h_{1}$. Thus, there remains nothing to show.

$k>1$ : Suppose the statement is true for $k-1$. Note that $M_{h_{k-1}}(i) \neq M_{h_{0}}(i)$ and $h_{k-1} \succ^{i} h_{0}$ implies $M_{h_{k-1}}(i) \hat{\succ}^{i} M_{h_{0}}(i)$. Let $c_{k-1} \in M$ be the group and $D_{k-1} \in \mathcal{D}_{h_{k-1}}^{c_{k-1}}$ be the subset of players with $h_{k}=h_{k-1} \pm\left(c_{k-1}, D_{k-1}\right)$. First consider the case $i \in D_{k-1}$. By assumption every player $j \in D_{k-1}$ strictly benefits from the deviation. Because preferences are lexicographic, this implies not only $h_{k} \succ^{i} h_{0}$ but also $M_{h_{k}}(i) \neq M_{h_{0}}(i)$. Now suppose $i \notin D_{k-1}$. Then, of course, $M_{h_{k}}(i)=M_{h_{k-1}}(i) \neq M_{h_{0}}(i)$. But it might be possible that $i$ suffers from this deviation, i.e., $h_{k-1} \succ^{i} h_{k}$. Nevertheless, because $M_{h_{k}}(i)=M_{h_{k-1}}(i) \hat{\succ}^{i} M_{h_{0}}(i)$ the player still strictly prefers $h_{k}$ to $h_{0}$.

Additional lemma for the proofs. Some of the following proofs use similar technical arguments and the following lemma will serve as a convenient and useful tool. Recall that for each $h \in \mathcal{H}$ and $c \in M, \mathcal{A}_{h}^{c}(\mathcal{C})=\left\{D \in \mathcal{D}_{h}^{c} \mid \exists S \in \mathcal{S}_{h}^{c}(D)\right.$ such that $\left.h \pm(c, D) \succ^{i} h \forall i \in(D \backslash h(c)) \cup S\right\}$ is the set of all feasible deviations that are not blocked. That is, the feasible deviations that strictly benefit all the deviating players joining the group and all members of at least one of the supporting coalitions. We say that $h$ is exit-proof if $D \in \mathcal{A}_{h}^{c}(\mathcal{C})$ implies $D \nsubseteq h(c)$ for all $c \in M$. In other words, in an exit-proof group structure, no group of players wants or is forced to leave a group. Phrased differently, a group structure $h$ is not exit-proof if and only if there exists a group $c \in M$ and a coalition of members $D \subseteq h(c)$ which is forced to leave the group (i.e., $D \in \mathcal{A}_{h}^{c}(\mathcal{C})$ ).

Lemma 2. Let $(N, M, \succeq, \mathcal{C})$ be a society. Moreover, let $h \in \mathcal{H}$ be an arbitrary group structure. Then there exists an exit-proof group structure $\bar{h} \in I(h)$.

Proof. Let $c \in M$ such that there exists $D \in \mathcal{A}_{h}^{c}$ with $D \subseteq h(c)$. If such a group does not exist, the group structure is exit-proof already and there remains nothing to show. Consider $h^{\prime}:=h-(c, D)$. If $h^{\prime}(c)$ is not exit-proof, further subsets of players can be forced to leave $c$ until the group does not contain any member or no 
coalition is supporting these deviations any more. This proceeding can be repeated for all groups and because $N$ and $M$ are finite, after finitely many steps an exit-proof group structure $\bar{h}$ will be reached.

Using this lemma, Lemma 1 could be restated as follows. Lemma 1: Let the society $(N, M, \succeq, \mathcal{C})$ be given. There exists no closed cycle if and only if, for each exit-proof group structure $\bar{h} \in \mathcal{H}$ that is not constitutionally stable, there exists an improving path leading from $\bar{h}$ to a constitutionally stable group structure.

Proof of Proposition 4. The main idea of the proof is to construct for every group structure in $H$ an improving path leading from this group structure to a constitutionally stable group structure. By closedness, this stable group structure is in $H$, too. Hence, there cannot be a closed cycle in $H$.

For constructing these paths, let us define, for each group structure $h \in H$, the set

$$
\bar{M}_{h}=\left\{c \in M \mid \exists j \in h(c): h \succ^{j} h-(c,\{j\})\right\} .
$$

That is, a group $c \in M$ is contained in $\bar{M}_{h}$ if and only if at least one of its members does not want to leave $c$. In particular, if $c \in \bar{M}_{h}$, due to equability, none of the members wants to leave $c$.

Let $h_{1} \in H$ be an arbitrary group structure. By applying Lemma 2 we may assume that $h_{1}$ is exit-proof. In the following, we will establish that if $h_{1}$ is not constitutionally stable (if this would be the case, there would remain nothing to be shown), there exists an improving path from $h_{1}$ to another exit-proof group structure $h_{2}$ such that either $\bar{M}_{h_{1}} \subsetneq \bar{M}_{h_{2}}$ or $\bar{M}_{h_{1}}=\bar{M}_{h_{2}}$ and $h_{1} \subsetneq h_{2}$. Then, if $h_{2}$ is not constitutionally stable, it is possible to iterate the previous step again and again. In particular, each time the step is iterated, either there are more groups whose members do not want to leave or the group structure strictly grows. Since both, the set of groups and the set of players, are supposed to be finite, this procedure will end after finitely many steps.

Case 1: There exists $c \in M \backslash \bar{M}_{h_{1}}$ with $\mathcal{A}_{h_{1}}^{c} \neq \emptyset$.

Note that because $h_{1}$ is exit-proof, $D \in \mathcal{A}_{h_{1}}^{c}$ if and only if $D \nsubseteq h_{1}(c)$, i.e., there is at least one player $i_{1} \in D \backslash h_{1}(c)$ who joins the group. Let $h_{1}^{\prime}:=h_{1} \pm(c, D)$. Because all players are self-concerned, this implies:

$$
h_{1} \sim^{i_{1}} h_{1} \pm\left(c, D \backslash\left\{i_{1}\right\}\right)=h_{1}^{\prime}-\left(c,\left\{i_{1}\right\}\right)
$$

In other words, after joining the group, player $i_{1}$ has no incentive to leave it unilaterally. By equability this is also true for all $i \in h_{1}^{\prime}(c)$ and, thus, $c \in \bar{M}_{h_{1}^{\prime}}$. Now let $c^{\prime} \in \bar{M}_{h_{1}}$. Note that $c \neq c^{\prime}$ and $h_{1}\left(c^{\prime}\right)=h_{1}^{\prime}\left(c^{\prime}\right)$. Therefore, equability implies that 
$c^{\prime} \in \bar{M}_{h_{1}^{\prime}}$, too. Moreover, assume there exists $D^{\prime} \in \mathcal{A}_{h_{1}^{\prime}}^{c^{\prime}}$ with $D^{\prime} \subseteq h_{1}^{\prime}\left(c^{\prime}\right)$, that is, assume that $c^{\prime}$ is not exit-proof any more. Let $S^{\prime} \in \mathcal{S}_{h_{1}^{\prime}}^{c^{\prime}}\left(D^{\prime}\right)$ be the corresponding supporting coalition. From regularity, it follows that there is a player $j \in S^{\prime}$ with $h_{1} \succeq^{j} h_{1}-\left(c^{\prime}, D^{\prime}\right)$ but $h_{1}^{\prime}-\left(c^{\prime}, D^{\prime}\right) \succeq^{j} h_{1}^{\prime}$. If $j \notin D^{\prime}$, this would contradict separability because $h_{1}\left(c^{\prime}\right)=h_{1}^{\prime}\left(c^{\prime}\right)$. If $j \in D^{\prime}$, this would violate equability and self-concern. Therefore, the assumption cannot be true or, in other words, transforming $c$ does not affect the exit-proofness of $c^{\prime}$. Similar considerations also apply if $c^{\prime} \in M \backslash \bar{M}_{h_{1}}$ with $c^{\prime} \neq c$. However, it might be possible that $c$ itself is not exit-proof any more. In this case, we can delete (analogously to Lemma 2) all coalitions of players from the group under the conditions that (i) no player joins $c$ and (ii) all deviations comply with the constitutions, i.e., they are feasible and supported by a supporting coalition. Let $h_{2}$ be the group structure which is finally reached by means of this procedure. In particular, by advancing the same arguments as before it can be shown that the other groups are still exit-proof in $h_{2}$ and, moreover, $\bar{M}_{h_{1}}=\bar{M}_{h_{2}} \backslash\{c\} \nsubseteq \bar{M}_{h_{2}}$.

Case 2: $\mathcal{A}_{h_{1}}^{c}=\emptyset$ for all $c \in M \backslash \bar{M}_{h_{1}}$.

Because $h_{1}$ is not constitutionally stable, there exists $c_{1} \in \bar{M}_{h_{1}}$ with $\mathcal{A}_{h_{1}}^{c_{1}} \neq \emptyset$. Let $D \in \mathcal{A}_{h_{1}}^{c_{1}}$ be of minimal size, i.e., $\tilde{D} \notin \mathcal{A}_{h_{1}}^{c_{1}}$ for all $\tilde{D} \subsetneq D$. Moreover, let $S \in \mathcal{S}_{h_{1}}^{c_{1}}(D)$ be the corresponding coalition which supports the deviation of $D$. We will show first that $D \cap h_{1}\left(c_{1}\right)=\emptyset$, that is, there are only players in $D$ who join the group $c$. Assume this is not true, i.e., there exists $i \in D \cap h_{1}\left(c_{1}\right)$. Then, $h_{1} \succ^{i} h_{1}-\left(c_{1},\{i\}\right) \sim^{i} h_{1} \pm\left(c_{1}, D\right)$ by self-concern and the definition of $\bar{M}_{h_{1}}$. Thus, $i$ would not support the deviation of $D$. Expressed differently, $S \cap D=\emptyset$. Because all constitutions are supposed to be decomposable and regular, we also have $\{i\} \in \mathcal{D}_{h_{1}}^{c_{1}}$ and $S \in \mathcal{S}_{h_{1}}^{c_{1}}(\{i\})$. By construction of $h_{1}$ the group structure is exit-proof and, therefore, there exists a player $j \in S$ with $h_{1} \succeq^{j} h_{1}-\left(c_{1},\{i\}\right)$. In particular, due to uniformity this is true for all members of $S$. But exploiting separability then yields $h_{1} \pm\left(c_{1}, D \backslash\{i\}\right) \succeq^{j} h_{1} \pm\left(c_{1}, D\right) \succ^{j} h_{1}$ for all $j \in S$ which contradicts the minimality of $D$.

Define $h_{2}:=h_{1}+\left(c_{1}, D\right)$. Because all $i \in D$ agreed to joining $c_{1}, h_{2} \succ^{i} h_{1} \sim^{i} h_{2}-$ $\left(c_{1},\{i\}\right)$ by self-concern. Therefore, from equability it follows that no player in $h_{2}\left(c_{1}\right)$ wants to leave the group unilaterally. Moreover, if $\bar{D} \in \mathcal{D}_{h_{2}}^{c_{1}}$ with $\bar{D} \cap h_{2}\left(c_{1}\right) \neq \emptyset$, then

$$
h_{2} \succ^{i} h_{2}-\left(c_{1},\{i\}\right) \sim^{i} h_{2} \pm\left(c_{1}, \bar{D}\right)
$$

for all $i \in \bar{D} \cap h_{2}\left(c_{1}\right)$, again by self-concern. In other words, all players who would have to leave the group would suffer from this deviation.

In the remainder of the proof we will show that $h_{2}$ is indeed exit-proof. Let $c^{\prime} \in M$ be an arbitrary group and $D^{\prime} \in \mathcal{D}_{h_{2}}^{c^{\prime}}$ with $D^{\prime} \subseteq h_{2}\left(c^{\prime}\right)$. Recall that $\mathcal{D}_{h_{2}}^{c^{\prime}}=\mathcal{D}_{h_{1}}^{c^{\prime}}$ by regularity and, thus, $D^{\prime} \in \mathcal{D}_{h_{1}}^{c^{\prime}}$, too. 
First consider the case $c_{1} \neq c^{\prime}$. Since the agents' preferences are separable, $h_{2} \succeq^{j} h_{2}-\left(c^{\prime}, D^{\prime}\right)$ if and only if $h_{1} \succeq^{j} h_{1}-\left(c^{\prime}, D^{\prime}\right)$ for all $j \in h_{2}\left(c^{\prime}\right) \backslash D^{\prime}$. Therefore, if $j \in h_{2}\left(c^{\prime}\right) \backslash D^{\prime}$ does not support the deviation of $D^{\prime}$ in $h_{1}$, the same holds for $h_{2}$, too. However, this is also true for all $j \in D^{\prime}$ due to equability and self-concern. Hence, it follows that a coalition supports a deviation in $h_{2}$ if and only if it does the same in $h_{1}$ (cf. Case 1). In particular, this implies that the group $c^{\prime}$ is also exit-proof in $h_{2}$.

Next consider $c^{\prime}=c_{1}$. Here we have to distinguish two cases, $S=\emptyset$ and $S \neq \emptyset$. First consider $S=\emptyset$, that is, when deviating from $h_{1}$ to $h_{2}$, the agents in $D$ do not need the consent of other members for entering $c$. Assume there exists $D^{\prime} \in \mathcal{A}_{h_{2}}^{c_{1}}$ with $D^{\prime} \subseteq h_{2}\left(c_{1}\right)$. Let $S^{\prime} \in \mathcal{S}_{h_{2}}^{c_{1}}\left(D^{\prime}\right)$ be a coalition which supports the deviation of $D^{\prime}$, i.e., there is no $j \in S^{\prime}$ with $h_{2} \succeq^{j} h_{2}-\left(c_{1}, D^{\prime}\right)$. From Equation (3) follows $D^{\prime} \cap S^{\prime}=\emptyset$. Moreover, regularity implies that there exists $\emptyset \neq S^{\prime \prime} \in \mathcal{S}_{h_{1}}^{c_{1}}\left(D^{\prime}\right)$ with $S^{\prime \prime} \subseteq S^{\prime}$. Note that $h_{2}-\left(c_{1}, D^{\prime}\right)=\left(h_{1}+\left(c_{1}, D\right)\right)-\left(c_{1}, D^{\prime}\right)=h_{1} \pm\left(c_{1}, D \pm D^{\prime}\right)$. In particular, $D^{\prime} \subseteq h_{1}\left(c_{1}\right)$ if and only if $D \cap D^{\prime} \neq \emptyset$. However, this is not possible because this would contradict separability of the players' preferences. Therefore, $D \cap D^{\prime} \neq \emptyset$. But this is not possible, too: by decomposability and regularity also $D \cap D^{\prime} \in \mathcal{D}_{h_{2}}^{c_{1}} \subseteq \mathcal{D}_{h_{1}}^{c_{1}}$ and $S^{\prime} \in \mathcal{S}_{h_{2}}^{c_{1}}\left(D \cap D^{\prime}\right)$. Because $\emptyset \in \mathcal{S}_{h_{1}}^{c_{1}}\left(D \cap D^{\prime}\right)$, again decomposability and regularity implies $D \cap D^{\prime} \subseteq S^{\prime}$ which contradicts Equation (3). Next consider $S \neq \emptyset$. We will show that $|D|=1$. Let $i \in D$. If there would be no player $j \in S$ with $h_{1}+(c,\{i\}) \succ^{i} h_{1}$, decomposability together with separability would imply $h_{1}+(c, D \backslash\{i\}) \succeq^{j} h_{1}+(c, D)=h_{2} \succ^{j} h_{1}$ for all $j \in S$. In other words, $S$ would also support a deviation of $D \backslash\{i\}$. Moreover, from uniformity it follows $h_{1}+(c, D \backslash\{i\}) \succeq^{j} h_{1}+(c, D)=h_{2} \succ^{j} h_{1}$ for all $j \in h_{1}(c) \cup(D \backslash\{i\})$. Thus, the players in $D \backslash\{i\}$ would agree to joining $c$ without player $i$ which would contradict minimality of $D$. Therefore, given that each $i \in D$ is supported by at least one player in $S$, from uniformity it follows that this is also true for all other members of $h_{1}\left(c_{1}\right)$. That is, $h_{1}+\left(c_{1},\{i\}\right) \succ^{j} h_{1}$ for all $j \in h_{1}\left(c_{1}\right)$ and, thus, $h_{1}+\left(c_{1},\{i\}\right) \succ^{j} h_{1} \succ^{j} h_{1}-\left(c_{1},\{j\}\right) \sim^{j}\left(h_{1}+\left(c_{1},\{i\}\right)\right)-\left(c_{1},\{j\}\right)$ because $c_{1} \in \bar{M}_{h_{1}}$. By equability this also holds for player $i$ or, phrased differently, $i$ has an incentive for joining $c_{1}$ unilaterally. In fact, this implies $D=\{i\}$ by minimality of $D$. Moreover, by uniformity, all players in $h_{1}\left(c_{1}\right)$ strictly benefit from deviating from $h_{1}$ to $h_{2}$. Now let $D^{\prime}, S^{\prime}$, and $S^{\prime \prime}$ be given as in the case $S=\emptyset$. Then, as before we have $D^{\prime} \cap D \neq \emptyset$ and, thus, $i \in D^{\prime}$. By decomposability also $\left(h_{1}\left(c_{1}\right) \cap\left(D \pm D^{\prime}\right)\right)=h_{1}\left(c_{1}\right) \cap D^{\prime} \in \mathcal{D}_{h_{1}}^{c_{1}}$ and $S^{\prime \prime} \in \mathcal{S}_{h_{1}}^{c_{1}}\left(h_{1}\left(c_{1}\right) \cap D^{\prime}\right)$. Since we have $\bar{D} \in \mathcal{A}_{h_{1}}^{c_{1}}$ only if $\bar{D} \nsubseteq h_{1}\left(c_{1}\right)$, there exists $j \in S^{\prime \prime}$ with $h_{1} \succeq^{j} h_{1}-\left(c_{1}, h_{1}\left(c_{1}\right) \cap D^{\prime}\right)$. But this implies:

$$
h_{1}-\left(c_{1}, h_{1}\left(c_{1}\right) \cap D^{\prime}\right)=h_{2}-\left(c_{1}, D^{\prime}\right) \succeq^{j} h_{2} \succ^{j} h_{1} \succeq^{j} h_{1}-\left(c_{1}, h_{1}\left(c_{1}\right) \cap D^{\prime}\right)
$$

which obviously is a contradiction. Thus, the assumption $D^{\prime} \subseteq h_{2}\left(c_{1}\right), D^{\prime} \in \mathcal{A}_{h_{1}}^{c_{1}}$ 
must be false and $c_{1}$ is also exit-proof in $h_{2}$.

Proof of Proposition 5. The proof proceeds in a similar way as the one of Proposition 4. As above we will construct for every exit-proof group structure $h_{1} \in H$ an improving path leading to a constitutionally stable group structure.

Step 1: In this step we establish that if $h_{1}$ is not constitutionally stable, there exists an improving path to another exit-proof group structure $h_{2}$ such that there is $D_{1} \subseteq N$ with $h_{2} \succ^{i} h_{1}$ and $M_{h_{1}}(i) \neq M_{h_{2}}(i)$ for all $i \in D_{1}$. Note that this implies $h_{1} \neq h_{2}$. Therefore, suppose $h_{1}$ is not constitutionally stable. Then there exists $c_{1} \in M$ with $\mathcal{A}_{h_{1}}^{c_{1}} \neq \emptyset$. Let $D_{1} \in \mathcal{A}_{h_{1}}^{c_{1}}$ be of minimal size, i.e., $\tilde{D} \notin \mathcal{A}_{h_{1}}^{c_{1}}$ for all $\tilde{D} \subsetneq D_{1}$. Moreover, let $S \in \mathcal{S}_{h_{1}}^{c_{1}}\left(D_{1}\right)$ be the corresponding coalition which supports the deviation of $D_{1}$. We will show first that $\left|D_{1}\right|=1$. Note that $D_{1} \nsubseteq h_{1}\left(c_{1}\right)$ because $h_{1}$ is exit-proof by assumption. Moreover, for all $i \in D_{1}$ there is at least one $j \in S$ with $h_{1}+\left(c_{1},\{i\}\right) \succ^{j} h_{1}$. If this would not be satisfied, analogously to Case 2 in the proof of Proposition 4 we would have $D_{1} \backslash\{i\} \in \mathcal{A}_{h_{1}}^{c_{1}}$ since the constitutions are decomposable and the preferences are separable and lexicographic. But this would contradict minimality of $D_{1}$. Therefore, given that each $i \in D_{1}$ is supported by at least one player in $S$, from uniformity it follows that this also holds for all other members of $h_{1}\left(c_{1}\right)$ and, thus, $D_{1}=\{i\}$ by minimality of $D_{1}$. Moreover, by applying uniformity, all members in $h_{1}\left(c_{1}\right)$ are strictly better off if $i$ enters the group. Next we show that $c_{1}$ is also exit-proof in $\bar{h}:=h_{1}+\left(c_{1},\{i\}\right)$. Assume this is not true, that is, assume there exists $D^{\prime} \in \mathcal{A}_{\bar{h}}^{c_{1}}$ with $D^{\prime} \subseteq \bar{h}\left(c_{1}\right)$. Analogously to Case 2 in the proof of Proposition 4 we must have $i \in D^{\prime}$ because the players's preferences are lexicographic and separable. Let $S^{\prime} \in \mathcal{S}_{\bar{h}}^{c_{1}}\left(D^{\prime}\right)$ be a coalition which supports the deviation of $D^{\prime}$. Moreover, let $S^{\prime \prime} \in \mathcal{S}_{h_{1}}^{c_{1}}\left(D^{\prime}\right)$ with $S^{\prime \prime} \subseteq S^{\prime}$ be defined as in Case 2 in the proof of Proposition 4. Then, by advancing analog arguments as above we get

$$
h_{1}-\left(c_{1}, h_{1}\left(c_{1}\right) \cap D^{\prime}\right)=\bar{h}-\left(c_{1}, D^{\prime}\right) \succ^{j} \bar{h} \succ^{j} h_{1} \succeq^{j} h_{1}-\left(c_{1}, h_{1}\left(c_{1}\right) \cap D^{\prime}\right)
$$

which obviously is a contradiction. Thus, the assumption $D^{\prime} \subseteq \bar{h}\left(c_{1}\right), D^{\prime} \in \mathcal{A}_{h_{1}}^{c_{1}}$ must be false and $c_{1}$ is also exit-proof in $\bar{h}$.

Now, suppose there exists $c^{\prime} \neq c_{1}$ with $\bar{D} \in \mathcal{A}_{\bar{h}}^{c^{\prime}}$ for some $\bar{D} \subseteq \bar{h}\left(c^{\prime}\right)=h_{1}\left(c^{\prime}\right)$. Let $\bar{S} \in \mathcal{S}_{\bar{h}}^{c^{\prime}}(\bar{D})$ be the corresponding supporting coalition. Note that $\bar{D} \cap \bar{S} \neq \emptyset$ because of separability. Let $i \in \bar{D} \backslash \bar{S}$. By decomposability and regularity also $\{i\} \in \mathcal{D}_{\bar{h}}^{c^{\prime}}=\mathcal{D}_{h_{1}}^{c^{\prime}}$ and $\bar{S} \in \mathcal{S}_{\bar{h}}^{c^{\prime}}(\{i\})$. Since $h_{1}$ is exit-proof, there exists $j \in \bar{S}$ with $h_{1} \succeq^{j} h_{1}-\left(c^{\prime},\{i\}\right)$ and, thus, also $\bar{h} \succeq^{j} \bar{h}-\left(c^{\prime},\{i\}\right)$. Therefore, because the players' preferences satisfy uniformity, $\bar{h} \succeq^{\bar{j}} \bar{h}-\left(c^{\prime},\{i\}\right)$ for all $\bar{j} \in \bar{h}\left(c^{\prime}\right) \backslash\{i\}$. By exploiting separability this yields

$$
\bar{h}-\left(c^{\prime}, \bar{D} \backslash\{i\}\right) \succeq^{\bar{j}}\left(\bar{h}-\left(c^{\prime}, \bar{D} \backslash\{i\}\right)\right)-\left(c^{\prime},\{i\}\right)=\bar{h}-\left(c^{\prime}, \bar{D}\right) \succ^{\bar{j}} \bar{h}
$$


for all $\bar{j} \in \bar{S}$. Iterating this argument implies $\bar{D} \cap \bar{S} \in \mathcal{A}_{\bar{h}}^{c^{\prime}}$, too, and $\bar{D} \backslash \bar{S} \notin \mathcal{A} \mathcal{A}_{\bar{h}}^{\prime}$. Therefore, all players in $\bar{D} \cap \bar{S} \in \mathcal{A}_{\bar{h}}^{c^{\prime}}$ strictly benefit from this deviation. Note that it might be the case that there exists $j \in \bar{h}\left(c^{\prime}\right) \cap D$ who is worse off after this change of the group. However, because the preferences are lexicographic, this player still strictly prefers $\bar{h}-(c, \bar{D} \cap \bar{S})$ to $h_{1}$. By iterating these arguments all subsets of members where all players agree to deviate can be deleted from all groups. Let $h_{2}$ be the group structure which is finally reached by means of this procedure. In particular, because of separability and uniformity, $h_{2}$ is exit-proof, too. Moreover, since no player has to leave a group against her will and preferences are lexicographic, all players who deviated strictly prefer $h_{2}$ to $h_{1}$.

Step 2: In this step we show that if $h_{2}$ is not stable, there exists

(i) a sequence of non-empty subsets $D_{1}, D_{2}, \ldots, D_{k-1}$, and

(ii) a sequence of exit-proof group structures $h_{1}, h_{2}, h_{3}, \ldots, h_{k}$ such that there is an improving path from $h_{l-1}$ to $h_{l}$ for all $2 \leq l \leq k$ and the following two conditions are satisfied:

(a) $h_{l} \succ^{i} h_{l^{\prime}}$ for all $2 \leq l \leq k, 1 \leq l^{\prime} \leq l-1$, and $i \in D_{l-1}$;

(b) if $h_{l} \nsucc^{i} h_{l-1}$, then $M_{h_{l}}(i)=M_{h_{l-1}}(i)$.

In particular, (a) implies $h_{k} \neq h_{l^{\prime}}$ for all $1 \leq l^{\prime}<k$. Therefore, since there are only finitely many exit-proof group structures, this sequence will stop after finitely many steps and, thus, the last one has to be constitutionally stable.

We will show the existence of the sequence by means of induction. For $k=2$ see Step 1. Consequently, let $k \geq 3$ and assume there exist $h_{3}, \ldots, h_{k}$ and $D_{2}, \ldots, D_{k-1}$ as defined above. Moreover, suppose $h_{k}$ is not stable. Since this group structure is exit-proof by assumption, there exists $c_{k} \in M$ with $\mathcal{A}_{h_{k}}^{c_{k}} \neq \emptyset$ and $D \nsubseteq h_{k}\left(c_{k}\right)$ for all $D \in \mathcal{A}_{h_{k}}^{c_{k}}$. Let $D_{k} \in \mathcal{A}_{h_{k}}^{c_{k}}$ be of minimal size and construct $h_{k+1}$ analogously to $h_{2}$ in Step 1. Similar to above, players deviate only if they have a strict incentive and $h_{k+1} \succ^{i} h_{k}$ for all $i \in D_{k}$. First, this implies $M_{h_{k}}(i)=M_{h_{k+1}}(i)$ for all $i \in N$ with $h_{k+1} \chi^{i} h_{k}$. Second, if $i \in D_{k} \cap D_{k-i}$, then clearly $h_{k+1} \succ^{i} h_{l^{\prime}}$ for all $1 \leq l^{\prime} \leq k$ because of transitivity. Therefore, let $i \in D_{k+1} \backslash D_{k}$. If $M_{h_{k}}(i)=M_{h_{l^{\prime}}}(i)$ for all $1 \leq l^{\prime} \leq k$, we have $h_{k+1} \succ^{i} h_{l^{\prime}}$ for each of these group structures because $i$ 's preferences are lexicographic. On the other hand, if $M_{h_{k}}(i) \neq M_{h_{1}}(i)$, let $l_{1}:=$ $\min \left\{2 \leq l \leq k \mid M_{h_{l-1}}(i) \neq M_{h_{l}}(i)\right\}$. Note that (ii) implies $h_{l_{1}} \succ^{i} h_{l_{1}-1}$. Thus, from this also follows $h_{l_{1}} \succ^{i} h_{l^{\prime}}$ for all $1 \leq l^{\prime} \leq l_{1}-1$ by lexicography. Next consider $l_{2}:=\min \left\{l_{1}+1 \leq l \leq k \mid M_{h_{l-1}}(i) \neq M_{h_{l}}(i)\right\}$. By advancing analog arguments as before we get $h_{l_{2}} \succ^{i} h_{l^{\prime}}$ for all $1 \leq l^{\prime} \leq l_{2}-1$ and, thus, iterating the procedure yields $h_{k+1} \succ^{i} h_{l^{\prime}}$ for all $1 \leq l^{\prime} \leq k$. 
Proof of Proposition 6. This proof proceeds similarly as the proofs of the two previous propositions. Again, we construct for every firm structure which is in $H=\left\{h \in \mathcal{H} \mid O \cap h(c)=\left\{o_{c}\right\} \forall c \in M\right\}$ an improving path leading from this firm structure to a constitutionally stable firm structure. Because $H$ is closed, this stable firm structure has to be in $H$, too. Therefore, let $h_{1} \in H$ be an arbitrary firm structure. Because of Lemma 2 we may assume that $h_{1}$ is exit-proof. Moreover, let $c_{1} \in M$ be an arbitrary firm with $\mathcal{A}_{h_{1}}^{c_{1}} \neq \emptyset$. The construction of the path proceeds in three steps.

Step 1: We establish that there exists $B_{1} \in \mathcal{A}_{h_{1}}^{c_{1}}$ with $\mathcal{A}_{h_{1}+\left(c_{1}, B_{1}\right)}^{c_{1}}=\emptyset$.

The main idea of this step is to exploit separability of the owner's preferences. Let

$$
B_{1}:=\left\{i \in E \backslash h_{1}\left(c_{1}\right) \mid h_{1}+\left(c_{1},\{i\}\right) \succ^{i} h_{1} \text { and } h_{1}+\left(c_{1},\{i\}\right) \succ^{o_{c_{1}}} h_{1}\right\} .
$$

That is, $B_{1}$ contains exactly those players who want to join $c_{1}$ and would be accepted by $o_{c_{1}}$. Let $i, j \in B_{1}$. Then, $h_{1}+\left(c_{1},\{i, j\}\right) \succ^{o_{c_{1}}} h_{1}+\left(c_{1},\{i\}\right) \succ^{o_{c_{1}}} h_{1}$ by separability of $o_{c_{1}}$ 's preferences. Iterating this argument implies $h_{1}+\left(c_{1}, B_{1}\right) \succ^{o_{c_{1}}} h_{1}$. Moreover, since the workers' preferences are lexicographic, also $h_{1}+\left(c_{1}, B_{1}\right) \succ^{i} h_{1}$ for all $i \in B_{1}$. Thus, $B_{1} \in \mathcal{A}_{h_{1}}^{c_{1}}$. Now suppose there exists $D \in \mathcal{A}_{h_{1}+\left(c_{1}, B_{1}\right)}^{c_{1}}$. If $D \subseteq h_{1}\left(c_{1}\right) \cup B_{1}$, the definition of $B_{1}$ and exit-proofness of $h_{1}$ imply $h_{1}+\left(c_{1}, B_{1}\right) \succeq^{o_{1}}\left(h_{1}+\left(c_{1}, B_{1}\right)\right)-$ $\left(c_{1}, i\right)$ for all $i \in h_{1}\left(c_{1}\right) \cup B_{1}$. Advancing the same arguments as before yields $h_{1}+\left(c_{1}, B_{1}\right) \succeq^{o_{1}}\left(h_{1}+\left(c_{1}, B_{1}\right)\right)-\left(c_{1}, D\right)$, which implies that $o_{c_{1}}$ would not support the deviation. Moreover, the workers in $h_{1}\left(c_{1}\right) \cup B_{1}$ obviously do not want to leave the firm and thus, $D \subseteq h_{1}\left(c_{1}\right) \cup B_{1}$ cannot be true. However, if $D \nsubseteq h_{1}\left(c_{1}\right) \cup B_{1}$ and there exists $i \in D \backslash h_{1}\left(c_{1}\right)$ with $\left(h_{1}+\left(c_{1}, B_{1}\right)\right)+\left(c_{1},\{i\}\right) \succ^{o_{c_{1}}} h_{1}+\left(c_{1}, B_{1}\right)$, then by construction of $B_{1}$ and because $i$ 's preferences are lexicographic, this worker would not agree to join $c_{1}$. Therefore, $\mathcal{A}_{h_{1}+\left(c_{1}, B_{1}\right)}^{c_{1}}$ must be empty.

Step 2: We construct an improving path leading from $h_{1}^{\prime}:=h_{1}+\left(c_{1}, B_{1}\right)$ to another exit-proof firm structure $h_{2}$ with $h_{2} \succ^{i} h_{1}$ for all $i \in B_{1}$ and $h_{2} \succeq^{i} h_{1}$ for all $i \in E \backslash B_{1}$.

Let $c^{\prime} \in M$ be such that there exists $B^{\prime} \subseteq h_{1}^{\prime}\left(c^{\prime}\right)$ with $B^{\prime} \in \mathcal{A}_{h_{1}^{\prime}}^{c^{\prime}}$ and choose $B^{\prime}$ maximal with respect to "ᄃ", i.e., there exists no $\bar{B} \subseteq h_{1}^{\prime}\left(c^{\prime}\right)$ with $\bar{B} \in \mathcal{A}_{h_{1}^{\prime}}^{c^{\prime}}$ and $B^{\prime} \subsetneq \bar{B}$. Note that $c^{\prime} \neq c_{1}$ because $\mathcal{A}_{h_{1}^{\prime}}^{c_{1}}=\emptyset$. By assumption $o_{c^{\prime}}$ 's preferences are separable and, thus, $h_{1}^{\prime} \succeq^{o_{c^{\prime}}} h_{1}^{\prime}-\left(c^{\prime}, B^{\prime}\right)$ by exit-proofness of $h_{1}$. Therefore, $h_{1}^{\prime}-\left(c^{\prime}, B^{\prime}\right) \succ^{j} h_{1}^{\prime}$ for all $j \in B^{\prime}$. Now suppose there exists $i \in B^{\prime} \backslash B_{1}$. This implies $i \in h_{1}(c)$ if and only if $i \in h_{1}^{\prime}(c)$ for all $c \in M$. If $i$ has a strict incentive for leaving $c^{\prime}$ in $h_{1}^{\prime}$, she would also have a strict incentive for leaving the firm in $h_{1}$ because her preferences are lexicographic. But this contradicts exit-proofness of $h_{1}$ and, thus, $B^{\prime} \subseteq B_{1}$. Moreover, by construction of $B^{\prime}$ and separability of $o_{c^{\prime}}$ 's preferences, there exists no further set of workers $B^{\prime \prime} \subseteq h_{1}^{\prime}\left(c^{\prime}\right) \backslash B^{\prime}$ with $B^{\prime \prime} \in \mathcal{A}_{h_{1}^{\prime}-\left(c^{\prime}, B^{\prime}\right)}^{c^{\prime}}$. By iterating the previous procedure, it is possible to reach an exit-proof firm structure $h_{2}$ by 
deleting all workers from all firms they want to leave without impairing the other workers in $E \backslash B_{1}$. In particular, for all $i \in E \backslash B_{1}$ nothing changes and, therefore, they are indifferent between $h_{2}$ and $h_{1}$. However, all $i \in B_{1}$ strictly benefit from the deviations and thus, they strictly prefer $h_{2}$ to $h_{1}$.

Step 3: Iterating the procedure.

Once at $h_{2}$, if $A_{h_{2}}^{c}=\emptyset$ for all $c \in M$, there remains nothing to show. Therefore, assume there exists $c_{2} \in M$ with $A_{h_{2}}^{c_{2}} \neq \emptyset$. By repeating Steps 1 and 2 it is possible to find $B_{2} \subseteq E \backslash h_{2}\left(c_{2}\right)$ with $\mathcal{A}_{h_{2}+\left(c_{2}, B_{2}\right)}^{c_{2}}=\emptyset$ and to construct an improving path leading from $h_{2}+\left(c_{2}, B_{2}\right)$ to an exit-proof firm structure $h_{3}$. Analogously, $h_{2}$ will be Pareto dominated by $h_{3}$ from the workers' perspective. Because $H$ is finite, there exists only a finite number of exit-proof firm structures. Hence, this procedure will end after finitely many steps.

Proof of Proposition 7. If $\mathcal{S} \mathcal{T}^{\mathrm{PD}}=\mathcal{S} \mathcal{T}^{\mathrm{HF}}$, then also $h^{w o} \in \mathcal{S} \mathcal{T}^{\mathrm{HF}}$ because $h^{w o}$ is always stable in Protection against Dismissal and there remains nothing to show. For the other direction, suppose the statement is not true, i.e., $h^{w o} \in \mathcal{S} \mathcal{T}^{\mathrm{HF}}$ but $\mathcal{S T}^{\mathrm{HF}} \subsetneq \mathcal{S} \mathcal{T}^{\mathrm{PD}}$. Let $\bar{h} \in \mathcal{S} \mathcal{T}^{\mathrm{PD}} \backslash \mathcal{S} \mathcal{T}^{\mathrm{HF}}$. Then, there must be an owner $o_{c}$ who would block $\bar{h}$ if her degree of authority is strong enough, i.e., there exists an employee $i \in \bar{h}(c)$ such that $\bar{h}-(c,\{i\}) \succ^{o_{c}} \bar{h}$. Because $o_{c}$ 's preferences are separable and $h^{w o}$ is stable, this implies $i \notin h^{w o}(c)$. Otherwise, the owner would also have an incentive to dismiss the employee in $h^{w o}$. Thus, uniqueness of $h^{w o}$ yields that $i$ strictly prefers $h^{w o}$ to $h^{w o}+(c,\{i\})$. In particular, because her preferences are supposed to be strongly separable, she would also have a strict incentive for leaving firm $c$ at $\bar{h}$, but this contradicts the stability of this firm structure.

The proof of Proposition 8 proceeds analogously to the one of Proposition 7, just by reversing the role of owners and employees.

A.2. Common Ranking and Stability. We now examine alternative conditions under which the society will always induce a constitutionally stable group structure in the sense that, for every starting group structure, there is always an improving path leading to a constitutionally stable group structure.

Definition 8. Given the society $(N, M, \succeq, \mathcal{C})$, a common ranking $\unrhd$ is a complete and transitive ordering over $\mathcal{H}$ such that $D \in \mathcal{A}_{h}^{c}(\mathcal{C})$ implies $h \pm(c, D) \unrhd h$ for all $h \in \mathcal{H}$ and $c \in M$.

A common ranking $\unrhd$ reflects a certain level of consensus between the players in the sense that, whenever a feasible deviation $D$ from $h$ to an obtainable group structure $h \pm(c, D)$ is not blocked, then all players in the society agree that the resulting group structure $h \pm(c, D)$ should be ranked above $h$. The main idea is 
that the set of group structures can be decomposed into several equivalence classes and once a higher class is reached, this will not be reversed afterwards. Indeed, a deviation takes place only if the joining and supporting players agree that the resulting group structure is not contained in a lower class than the current one. Note that a priory this is not a restriction at all because it would be possible, for instance, to choose $\unrhd$ in such a way that all group structures are equivalent (i.e., $h \unrhd h^{\prime}$ as well as $h^{\prime} \unrhd h$ for all $h, h^{\prime} \in \mathcal{H}$ ). This immediately implies that a (not necessarily unique) common ranking always exists. However, the more consensus about beneficial deviations between the players, the stronger the restrictions that can be imposed by a common ranking. ${ }^{17}$

Proposition 9. Let the society $(N, M, \succeq, \mathcal{C})$ be given.

(i) There are no cycles if and only if there exists a common ranking $\unrhd$ such that for all $H \subseteq \mathcal{H}$ there is a unique $\unrhd$-maximal group structure $\hat{h} \in H$.

(ii) There are no closed cycles if and only if there exists a common ranking $\unrhd$ such that for all $h \in \mathcal{H}$ there is a unique $\unrhd$-maximal group structure $\hat{h} \in I(h)$.

Proof. (i) In order to show that existence of $\unrhd$ implies the non-existence of cycles, we will consider the counte-rposition of this statement. Therefore, assume there is a cycle $H \subseteq \mathcal{H}$. Since there exists a path from each group structure in $H$ to every other group structure in $H$, if $\unrhd$ is a common ranking, we must have $\bar{h} \unrhd \breve{h}$ as well as $\breve{h} \unrhd \bar{h}$ for all $\bar{h}, \breve{h} \in H$. Thus, there is no unique $\unrhd$-maximal element in $H$. For the other direction suppose there exists no cycle. The following algorithm proceeds in a similar way as the one in the proof of Theorem 1 in Jackson and Watts (2001). We start with the binary relation $\unrhd_{1}$ where $h \triangleright_{1} \bar{h}$ if and only if there exists an improving path from $\bar{h}$ to $h$. Because there is no cycle, $\unrhd_{1}$ is strict. Moreover, for all $h \in \mathcal{H}, c \in M$, and $D \in \mathcal{D}_{h}^{c}$, deviating from $h$ to $h \pm(c, D)$ always implies $h \pm(c, D) \triangleright_{1} h$ by construction. However, $\unrhd_{1}$ is not necessarily complete. Let $\tilde{h}, \breve{h} \in \mathcal{H}$ with neither $\tilde{h} \triangleright_{1} \breve{h}$ nor $\breve{h} \triangleright_{1} \tilde{h}$. We construct $\unrhd_{1}$ by adding $\tilde{h} \bar{\triangleright}_{1} \breve{h}$ to $\unrhd_{1}$, i.e., $h \unrhd_{1} \bar{h}$ if and only if $h \unrhd_{1} \bar{h}$ or $h=\tilde{h}$ and $\bar{h}=\breve{h}$. Moreover, let $\unrhd_{2}$ be the transitive closure of $\bar{\unrhd}_{1}$. We will show that $\unrhd_{2}$ still represents the preference profile

\footnotetext{
${ }^{17}$ Farrell and Scotchmer (1988) introduce the common ranking property that requires the existence of a linear ordering over all coalitions which coincides with any player's preference ordering over coalitions to which she belongs. A relaxed version of the common ranking property, the topcoalition property, is introduced by Banerjee et al. (2001) to guarantee the existence of a core partition. The common ranking that we introduce here is similar to the one of Farrell and Scotchmer (1988) and orders the group structures differing only in that a unique group $c$ of players has changed its composition according to the preferences of the joining and supporting players involved in the change of $c$.
} 
of the players, i.e., deviating from $h$ to $h \pm(c, D)$ always implies $h \pm(c, D) \triangleright_{2} h$ for all $c \in M$ and $D \in \mathcal{D}_{h}^{c}$. Suppose this is not true, that is, suppose there exist $h^{\prime} \in \mathcal{H}$, $c \in M, D \in \mathcal{D}_{h^{\prime}}^{c}$, and $S \in \mathcal{S}_{h^{\prime}}^{c}$ with $h^{\prime} \pm(c, D) \succ^{i} h^{\prime}$ for all $i \in\left(D \backslash h^{\prime}(c)\right) \cup S$ but $h^{\prime} \unrhd_{2} h \pm(c, D)$. Thus, there exists a sequence of group structures $\left(h_{0}, h_{1}, \ldots, h_{k}\right)$ with $h_{0}=h^{\prime}, h_{k}=h^{\prime} \pm(c, D)$ and $h_{0} \bar{\unrhd}_{1} h_{1} \bar{\unrhd}_{1} \ldots \bar{\unrhd}_{1} h_{k}$. Assume the sequence is of minimal length. This implies that $h_{l}=h_{l^{\prime}}$ only if $l=l^{\prime}$ for all $l, l^{\prime} \in\{0,1, \ldots, k\}$. Suppose there exists an $l \in\{1, \ldots, k\}$ with $\left\{h_{l-1}, h_{l}\right\}=\{\breve{h}, \tilde{h}\}$. Because $h_{l^{\prime}} \neq \breve{h}, \tilde{h}$ for all $l^{\prime} \notin\{l-1, l\}$ this yields

$$
h_{l} \unrhd_{1} h_{l+1} \unrhd_{1} \ldots \unrhd_{1} h_{k}=h^{\prime} \pm(c, D) \unrhd_{1} h^{\prime}=h_{0} \unrhd_{1} \ldots \unrhd_{1} h_{l-1}
$$

and, thus, there exists an improving path from $\tilde{h}$ to $\breve{h}$ or vice versa. This contradicts the assumption that the two group structures are not comparable under $\unrhd_{1}$. Therefore, there exists no $l \in\{1, \ldots, k\}$ with $\left\{h_{l-1}, h_{l}\right\}=\{\breve{h}, \tilde{h}\}$. From this follows $h_{0} \unrhd_{1} h_{1} \unrhd_{1} \ldots \unrhd_{1} h_{k}$ which contradicts the assumption that there is no cycle. Thus, $\unrhd_{2}$ still represents the preferences of the players and by construction it is also transitive and strict. If it is not complete, the previous steps can be iterated. Because the set of group structures is finite, the iteration will stop after finitely many steps and we obtain a common ranking $\unrhd$ which is strict. In particular, strictness implies that for each $H \subseteq \mathcal{H}$ there is a unique $\unrhd$-maximal group structure $\hat{h} \in H$.

(ii) The first direction proceeds analogously to the first direction of Part (i). Let a common ranking $\unrhd$ and a set of group structures $H \subseteq \mathcal{H}$ be given. If $H$ forms a closed cycle, we have $I(h)=I\left(h^{\prime}\right)=H$ and $h \unrhd h^{\prime}$ as well as $h^{\prime} \unrhd h$ for all $h, h^{\prime} \in H$. But this would contradict that there is a unique $\unrhd$-maximal group structure in $H$ and, thus, there cannot exist a closed cycle.

For the other direction suppose there exist no closed cycles. The first step of the construction of the common ranking proceeds in the same way as the one of Part (i). That is, we start with $\unrhd_{1}$ where $h \unrhd_{1} \bar{h}$ if and only if there exists an improving path from $\bar{h}$ to $h$. But note that here this binary relation is not necessarily strict. Since by assumption there are no closed cycles, there exists at least one constitutionally stable group structure $h^{\prime} \in \mathcal{H}$. If this group structure is uniquely determined, according to Lemma 1 it is contained in every closed subset $H \subseteq \mathcal{H}$ and $\unrhd_{1}$ can then obviously be extended to a complete ranking where $h^{\prime}$ is the unique maximal element. Therefore, in the following, suppose there exists a further constitutionally stable group structure $h^{\prime \prime} \in \mathcal{H}$. In particular, this implies that neither $h^{\prime} \unrhd_{1} h^{\prime \prime}$ nor $h^{\prime \prime} \unrhd_{1} h^{\prime}$. Let $\tilde{h}, \breve{h} \in \mathcal{H}$ be an arbitrary pair of group structures not comparable under $\unrhd_{1}$. Analogously to above, $\unrhd_{1}$ is constructed by adding $\tilde{h} \bar{\triangleright}_{1} \breve{h}$ to $\unrhd_{1}$, i.e., $h \bar{\unrhd}_{1} \bar{h}$ if and only if $h \unrhd_{1} \bar{h}$ or $h=\tilde{h}$ and $\bar{h}=\breve{h}$. Again, let $\unrhd_{2}$ be the transitive closure of $\unrhd_{1}$. Note that by construction $h^{\prime} \unrhd_{2} h^{\prime \prime}$ would imply $h^{\prime} \unrhd_{2} h^{\prime \prime}$ and vice versa. If $\unrhd_{2}$ is not complete, because of finiteness of $\mathcal{H}$ we can iterate the previous steps 
until a complete ranking $\unrhd$ is reached. We will show that $h^{\prime}$ and $h^{\prime \prime}$ are still not equivalent under $\unrhd$. This, in fact, has the following implication: If $\check{h}$ is $\unrhd$-maximal in a closed subset $H \subseteq \mathcal{H}$, it has to be constitutionally stable by construction and w.l.o.g. we may assume $\check{h}=h^{\prime}$. Then, for any other stable group structure $h^{\prime \prime} \in H$, we must have $h^{\prime} \triangleright h^{\prime \prime}$ and, thus, $h^{\prime}$ is the unique $\unrhd$-maximal element in $H$.

In order to show $h^{\prime}$ and $h^{\prime \prime}$ are still not equivalent under $\unrhd$, let $\unrhd_{k}$ be the binary relation constructed in the $k$-th step of the algorithm described in the previous passage. For $k=1,2$ we already know that $h^{\prime} \unrhd_{k} h^{\prime \prime}$ would imply $h^{\prime} \nsupseteq_{k} h^{\prime \prime}$ and vice versa. We will show inductively that this is also satisfied for all other $k$. Therefore, let $k \geq 3$ and suppose that $h^{\prime}$ and $h^{\prime \prime}$ are still not equivalent under $\unrhd_{k-1}$. Moreover, assume this is not satisfied under $\unrhd_{k}$, i.e., we have $h^{\prime} \unrhd_{k} h^{\prime \prime}$ as well as $h^{\prime \prime} \unrhd_{k} h^{\prime}$. This assumption will lead to a contradiction. Let $\tilde{h}^{(k-1)}, \breve{h}^{(k-1)} \in \mathcal{H}$ be the corresponding pair of group structures not comparable under $\unrhd_{k-1}$ which is added in the next step. We will distinguish three cases:

Case 1: $h^{\prime} \triangleright_{k-1} h^{\prime \prime}$.

Because we assume $h^{\prime}$ and $h^{\prime \prime}$ are not equivalent under $\unrhd_{k-1}$, this implies that there exists a sequence of group structures $\left(h_{1}, \ldots, h_{l}\right)$ with $h_{1}=h^{\prime \prime}, h_{l}=h^{\prime}$, and $h_{1} \bar{\unrhd}_{k-1} \ldots \bar{\unrhd}_{k-1} h_{l}$. Moreover, from this also follows that there exists $1 \leq l^{\prime} \leq l-1$ with $\left\{h_{l^{\prime}}, h_{l^{\prime}+1}\right\}=\left\{\tilde{h}^{(k-1)}, \breve{h}^{(k-1)}\right\}$. But then

$$
h_{l^{\prime}+1} \unrhd_{k-1} \ldots \unrhd_{k-1} h^{\prime} \triangleright_{k-1} h^{\prime \prime} \unrhd_{k-1} \ldots \triangleright_{k-1} h_{l^{\prime}}
$$

which contradicts that $\tilde{h}^{(k-1)}$ and $\breve{h}^{(k-1)}$ are not comparable under $\triangleright_{k-1}$.

Case 2: $h^{\prime \prime} \triangleright_{k-1} h^{\prime}$.

This case proceeds analogously to the previous one by just reversing the roles of $h^{\prime}$ and $h^{\prime \prime}$.

Case 3: $h^{\prime}$ and $h^{\prime \prime}$ are not comparable under $\unrhd_{k-1}$.

If $h^{\prime}$ and $h^{\prime \prime}$ are equivalent under $\unrhd_{k}$ but not under $\unrhd_{k-1}$, there must be two sequences of group structures $\left(h_{1}, \ldots, h_{l}\right)$ and $\left(\bar{h}_{1}, \ldots, \bar{h}_{\bar{l}}\right)$ with $h_{1}=\bar{h}_{\bar{l}}=h^{\prime}, h_{l}=\bar{h}_{1}=h^{\prime \prime}$, and

$$
h_{1} \bar{\unrhd}_{k-1} \ldots \bar{\unrhd}_{k-1} h_{l}=\bar{h}_{1} \bar{\unrhd}_{k-1} \ldots \bar{\unrhd}_{k-1} \bar{h}_{\bar{l}} .
$$

Moreover, there exist $1 \leq l^{\prime} \leq l-1$ and $1 \leq \bar{l}^{\prime} \leq \bar{l}-1$ with $\left\{h_{l^{\prime}}, h_{l^{\prime}+1}\right\}=\left\{\bar{h}_{\bar{l}^{\prime}}, \bar{h}_{\bar{l}^{\prime}+1}\right\}=$ $\left\{\tilde{h}^{(k-1)}, \breve{h}^{(k-1)}\right\}$. In particular, this yields

$$
h_{l^{\prime}} \unrhd_{k-1} h_{l^{\prime}+1} \unrhd_{k-1} \ldots \unrhd_{k-1} \quad h^{\prime \prime} \unrhd_{k-1} \ldots \unrhd_{k-1} \bar{h}_{\bar{l}^{\prime}} \unrhd_{k-1} \bar{h}_{\bar{l}^{\prime}+1}
$$

which could only be satisfied if $\tilde{h}^{(k-1)}$ and $\breve{h}^{(k-1)}$ are comparable under $\unrhd_{k-1}$.

The main importance of Proposition 9 is that it provides an alternative criterion for guaranteeing convergence to a constitutionally stable group structure. Item (i) 
states that requiring non-existence of cycles is equivalent to requiring the existence of a special common ranking which identifies a unique maximal element in every subset of group structures. A common ranking meets this requirement if and only if it is strict. In this case, it is a variation of "Generalized Ordinal Potentials" introduced by Monderer and Shapley (1996). In particular, item (i) of Proposition 3 is closely related to Lemma 2.5 from their publication. Moreover, it also relates to Theorem 1 in Jackson and Watts (2001). According to (ii), having this feature only in particular subsets of $\mathcal{H}$ is still strong enough for excluding closed cycles. Therefore, the society induces a constitutionally stable group structure for sure if and only if the constitutions allow for a common ranking which is sufficiently restrictive. That is, there must be some consent about which feasible deviations are beneficial and which are not.

A.3. Blocking Power and Stability. In our formulation, the constitutions grant the group members a certain level of blocking power allowing them to inhibit changes in the composition of the group that do not conform to their own preferences. Next proposition studies whether enhancing the blocking power of the individuals leads or not to more stability.

Proposition 10. Let two societies $(N, M, \succeq, \mathcal{C})$ and $(N, M, \succeq, \overline{\mathcal{C}})$ be given and assume that the constitution $\mathcal{C}$ restricts more the set of feasible deviations and the set of supporting coalitions than the constitution $\overline{\mathcal{C}}, \mathcal{C} \subseteq \overline{\mathcal{C}}$, i.e., $\mathcal{D}_{h}^{c} \subseteq \overline{\mathcal{D}}_{h}^{c}$ and $\mathcal{S}_{h}^{c}(D) \subseteq \overline{\mathcal{S}}_{h}^{c}(D)$ for all $h \in \mathcal{H}, c \in M$, and $D \in \mathcal{D}_{h}^{c}$. Then, the non-existence of closed cycles under $\overline{\mathcal{C}}$ does not imply that there are no closed cycles under $\mathcal{C}$ even if $\mathcal{S T}(\overline{\mathcal{C}}) \subseteq \mathcal{S T}(\mathcal{C})$

Proof. It is sufficient to construct a suitable example. The one we consider here is a variation of an example from Bogomolnaia and Jackson (2002). There are three players $N=\left\{i_{1} . i_{2}, i_{3}\right\}$ and one group $M=\{c\}$. Thus, $|\mathcal{H}|=8$. The group structures are given by:

\begin{tabular}{|c|cccccccc|}
\hline & $h_{1}(c)$ & $h_{2}(c)$ & $h_{3}(c)$ & $h_{4}(c)$ & $h_{5}(c)$ & $h_{6}(c)$ & $h_{7}(c)$ & $h^{\emptyset}$ \\
\hline$c$ & $\left\{i_{1}\right\}$ & $\left\{i_{2}\right\}$ & $\left\{i_{3}\right\}$ & $\left\{i_{1}, i_{2}\right\}$ & $\left\{i_{1}, i_{3}\right\}$ & $\left\{i_{2}, i_{3}\right\}$ & $\left\{i_{1}, i_{2}, i_{3}\right\}$ & $\emptyset$ \\
\hline
\end{tabular}

and the players' preferences are

$$
\begin{aligned}
& h_{4} \succ^{i_{1}} h_{7} \succ^{i_{1}} h_{5} \succ^{i_{1}} h_{1} \succ^{i_{1}} h_{2} \sim^{i_{1}} h_{3} \sim^{i_{1}} h_{6} \sim^{i_{1}} h^{\emptyset} \\
& h_{6} \succ^{i_{2}} h_{7} \succ^{i_{2}} h_{4} \succ^{i_{2}} h_{2} \succ^{i_{2}} h_{1} \sim^{i_{2}} h_{3} \sim^{i_{2}} h_{5} \sim^{i_{2}} h^{\emptyset} \\
& h_{5} \succ^{i_{3}} h_{7} \succ^{i_{3}} h_{6} \succ^{i_{3}} h_{3} \succ^{i_{1}} h_{1} \sim^{i_{3}} h_{2} \sim^{i_{3}} h_{4} \sim^{i_{3}} h^{\emptyset} .
\end{aligned}
$$

The setting is actually not completely the same as in Bogomolnaia and Jackson (2002), because in their paper the authors study coalition formation (i.e., the set of players is always decomposed into a partition) while we have just one group 
containing some of the players. However, "core stability" in their setting corresponds to constitutional stability with respect to the following constitution $\mathcal{C}^{c}=\left(\mathcal{D}^{c}, \mathcal{S}^{c}\right)$ :

$$
\mathcal{D}_{h}^{c}=2^{N} \backslash\{\emptyset\} \text { and } \mathcal{S}_{h}^{c}(D)=\{S \subseteq h(c) \mid(h(c) \backslash D) \subseteq S, S \neq \emptyset\}
$$

for all $h \neq h^{\emptyset}$. Given $\mathcal{C}^{c}$, a priory all possible deviating coalitions are feasible and a deviation $D \neq h(c)$ takes place if and only if all members of the resulting group structure benefit from the deviation, i.e., $h \pm(c, D) \succ^{i} h$ for all $i \in h(c) \pm D$. This implies that players who are undesired can be dismissed if the other members of the group agree on this. For the (pathological) special case of $D=h(c)$, it is required that at least one player approves the deviation. Now, given the constitution as defined in (1), we have that $h_{7}$ is the unique constitutionally stable (or "core stable", respectively) group structure and $H:=\left\{h_{4}, h_{6}, h_{5}\right\}$ forms a closed cycle. In fact, once $H$ is reached, there is no improving path leading to $h_{7}$ because the players act too myopically. However, consider the following constitutions $\overline{\mathcal{C}}^{c}=\left(\overline{\mathcal{D}}^{c}, \overline{\mathcal{S}}^{c}\right)$. Let $\overline{\mathcal{D}}_{h}^{c}=2^{N} \backslash\{\emptyset\}$ and

$$
\overline{\mathcal{S}}_{h}^{c}(D)= \begin{cases}\{S \subseteq h(c) \mid(h(c) \backslash D) \subseteq S, S \neq \emptyset\} & , \text { if } D \cap h(c) \neq \emptyset \\ \{S \subseteq h(c) \mid S \neq \emptyset\} & , \text { if } D \cap h(c)=\emptyset\end{cases}
$$

for all $h \neq h^{\emptyset}$. Here, granting access to $c$ to a coalition of players that are not member of the group just needs the support of only one member of the group. This obviously implies $\mathcal{C}^{c} \subsetneq \overline{\mathcal{C}}^{c}$ and, thus, the players have less blocking power under $\overline{\mathcal{C}}^{c}$ than under $\mathcal{C}^{c}$ (but note that the sets of constitutionally stable group structures coincide). However, under $\overline{\mathcal{C}}^{c}, H=\left\{h_{4}, h_{6}, h_{5}\right\}$ does not form a closed cycle any more because for all $h \in H$ there is always one member of $c$ who supports deviating from $h$ to $h_{7}$. Therefore, given $\overline{\mathcal{C}}^{c}$, there exists no closed cycle.

From the definition of constitutional stability we have that if the sets of feasible deviations and supporting coalitions shrink, the blocking power of each individual increases and the set of constitutionally stable group structures might become larger but never smaller. However, whether more blocking power really implies more stability, strongly depends on the adopted perspective of stability. Although the set of constitutionally stable group structures might become larger the greater the blocking power of the individuals, it could happen that the society will never reach one of these stable group structures because all improving paths leading to them could be destroyed and closed cycles could occur. 


\section{References}

[1] Acemoglu, D., Egorov, G. and K. Sonin, 2012. Dynamics and stability of constitutions, coalitions and clubs. American Economic Review 102(4), 1446-1476.

[2] Albizuri, M., Aurrecoechea, J. and J. Zarzuelo, 2006. Configuration values: Extensions of the coalitional Owen value. Games and Economic Behavior 57, $1-17$.

[3] Allouch, N. and M. Wooders, 2008. Price taking equilibrium in economies with multiple memberships in clubs and unbounded club sizes. Journal of Economic Theory 140, 246-278.

[4] Bala, V. and S. Goyal, 2000. A noncooperative model of network formation. Econometrica 68(5), 1181-1229.

[5] Banerjee, S., Konishi, H. and T.Sönmez, 2001. Core in a simple coalition formation game. Social Choice and Welfare 18, 135-153.

[6] Bloch, F., 1995. Endogenous structures of association in oligopolies. Rand Journal of Economics 26, 537-556.

[7] Bogomolnaia, A. and M.O. Jackson, 2002. The stability of hedonic coalition structures. Games and Economic Behavior 38, 201-230.

[8] Caulier, J.-F., Mauleon, A. and V. Vannetelbosch, 2013a. Contractually stable networks. International Journal of Game Theory 42, 483-499.

[9] Caulier, J.-F., Mauleon, A., Sempere-Monerris, J.J. and V. Vannetelbosch, 2013b. Stable and efficient coalitional networks. Review of Economic Design $17,249-271$.

[10] Cesco, J.C., 2012. Hedonic games related to many-to-one matching problems. Social Choice and Welfare 39(4), 737-749.

[11] Chalkiadakis, G., Elkind, E., Markakis, E., Polukarov, M. and N.R. Jennings, 2010. Cooperative games with overlapping coalitions. Journal of Artificial Intelligence Research 39, 179-216.

[12] Conconi, P. and C. Perroni, 2002. Issue linkage and issue tie-in in multilateral negotiations. Journal of International Economics 57, 423-447.

[13] Dang, V.D., Dash, R.K., Rogers, A. and N.R. Jennings, 2006. Overlapping coalition formation for efficient data fusion in multi-sensor networks. In Proc. of the 21st National Conference on AI (AAAI-06), 635-640. 
[14] Drèze, J.H. and J. Greenberg, 1980. Hedonic coalitions: optimality and stability. Econometrica 48(4), 987-1003.

[15] Dutta, B. and J. Masso, 1997. Stability of matchings when individuals have preferences over colleagues. Journal of Economic Theory 75, 464-475.

[16] Dutta, B. and S. Mutuswami, 1997. Stable networks. Journal of Economic Theory 76, 322-344.

[17] Echenique, F. and M.B. Yenmez, 2007. A solution to matching with preferences over colleagues. Games and Economic Behavior 59, 46-71.

[18] Ellickson, B., Grodal, B., Scotchmer, S. and W. Zame, 1999. Clubs and the market. Econometrica 67, 1185-1217.

[19] Ellickson, B., Grodal, B., Scotchmer, S. and W. Zame, 2001. Clubs and the market: Large finite economies. Journal of Economic Theory 101(1), 40-77.

[20] Farrell, J. and S. Scotchmer, 1988. Partnerships. The Quarterly Journal of Economics 103, 279-297.

[21] Fershtman, C. and D. Persitz, 2012. Clubs and social networks. Mimeo.

[22] Herings, P.J.J., Mauleon, A. and V. Vannetelbosch, 2009. Farsightedly stable networks. Games and Economic Behavior 67, 526-541.

[23] Jackson, M.O., 2008. Social and Economic Networks. Princeton University Press.

[24] Jackson, M.O. and A. Watts, 2001. The existence of pairwise stable networks. Seoul Journal of Economics 14(3), 299-322.

[25] Jackson, M.O. and A. Watts, 2002. The evolution of social and economic networks. Journal of Economic Theory 106, 265-295.

[26] Jehiel, P. and S. Scotchmer, 2001. Constitutional rules of exclusion in jurisdiction formation. The Review of Economic Studies 68, 393-413.

[27] Kelso, A.S. and V.P. Crawford, 1982. Job matching, coalition formation, and gross substitutes. Econometrica 50, 1483-1504.

[28] Kojima, F. and M.U. Unver, 2008. Random paths to pairwise stability in manyto-many matching problems: a study on market equilibration. International Journal of Game Theory 36, 473-488. 
[29] Kominers, S.D., 2010. Matching with preferences over colleagues solves classical matching. Games and Economic Behavior 68, 773-778.

[30] Mauleon, A., Sempere-Monerris, J.J. and V. Vannetelbosch, 2016. Contractually stable alliances. Journal of Public Economic Theory 18, 212-225.

[31] Myerson, R.B., 1980. Conference structures and fair allocation rules. International Journal of Game Theory 9(3), 169-182.

[32] Page, F.H. and M. Wooders, 2009. Strategic basins of attraction, the path dominance core, and network formation games. Games and Economic Behavior $66,462-487$.

[33] Page, F.H. and M. Wooders, 2010. Club networks with multiple memberships and noncooperative stability. Games and Economic Behavior 70, 12-20.

[34] Page, F.H., Wooders, M. and S. Kamat, 2005. Networks and farsighted stability. Journal of Economic Theory 120(2), 257-269.

[35] Pycia, M., 2012. Stability and preference alignment in matching and coalition formation. Econometrica 80(1), 323-362.

[36] Ray, D., 2007. A Game-Theoretic Perspective on Coalition Formation. Oxford: Oxford University Press.

[37] Ray, D. and R. Vohra, 2015. Coalition Formation. Chapter 5 of the Handbook of Game Theory, Volume 4. Edited by H.P. Young and S. Zamir. North-Holland, pp. 239-326.

[38] Roth, A.E., 1984. Stability and polarization of interests in job matching. Econometrica 52(1), 47-58.

[39] Roth, A.E., 1985. The college admissions problem is not equivalent to the marriage problem. Journal of Economic Theory 36, 277-288.

[40] Roth, A.E. and M. Sotomayor 1990. Two-sided Matching: A Study in GameTheoretic Modeling and Analysis. Cambridge University Press.

[41] Roth, A.E. and J.H. Vande Vate, 1990. Random paths to stability in two-sided matching. Econometrica 58, 1475-1480.

[42] Shenoy, O. and S. Kraus, 1996. Formation of overlapping coalitions for precedence-ordered task-execution among autonomous agents. In Proc. of the 2nd International Conference on Multi-Agent Systems (ICMAS-96), 330-337. 
[43] Sotomayor, M., 1999. Three remarks on the many-to-many stable matching problem. Mathematical Social Science 38, 55-70.

[44] Sotomayor, M., 2004. Implementation in the many-to-many matching market. Games and Economic Behavior 46, 199-212. 